\title{
DEUX SCARABÉES D'ORGAMÈ/ARGAMUM
}

\section{Anca Dan}

\section{Presses Universitaires de Franche-Comté | «Dialogues d'histoire ancienne »}

2011/1 37/1 | pages 9 à 40

ISSN 0755-7256

ISBN 97828486740

\section{Article disponible en ligne à l'adresse :}

https://www.cairn.info/revue-dialogues-d-histoire-ancienne-2011-1-page-9.htm

Distribution électronique Cairn.info pour Presses Universitaires de Franche-Comté.

(C) Presses Universitaires de Franche-Comté. Tous droits réservés pour tous pays.

La reproduction ou représentation de cet article, notamment par photocopie, n'est autorisée que dans les limites des conditions générales d'utilisation du site ou, le cas échéant, des conditions générales de la licence souscrite par votre établissement. Toute autre reproduction ou représentation, en tout ou partie, sous quelque forme et de quelque manière que ce soit, est interdite sauf accord préalable et écrit de l'éditeur, en dehors des cas prévus par la législation en vigueur en France. Il est précisé que son stockage dans une base de données est également interdit. 


\section{Deux scarabées d'Orgamè/Argamum}

Anca DAN*

La découverte des scarabées « égyptiens » en « faïence $»^{2}$ dans une $\pi o^{\lambda} ı s$ classique du Pont Gauche est un événement qui peut surprendre et intéresser à la fois le spécialiste de l'Égypte et celui de la Méditerranée grecque. Plusieurs questions surgissent dans l'esprit de l'historien et de l'archéologue à la vue de tels objets : où ont-ils été trouvés et que représentent-ils ? Quelle était leur signification originelle ? D'où viennent-ils, grâce à qui et à quelle époque ont-ils pu traverser, d'une extrémité à l'autre, le monde connu des Anciens ? Dans quelles circonstances ont-ils été acquis et adoptés par des habitants des bouches du Danube ? Pour quelle fonction ? En effet, une série assez importante de trouvailles faites, au cours des deux derniers siècles, dans tout l'œkoumène antique ${ }^{3}$ permet de parler d'une « égyptomanie » qui avait atteint

\footnotetext{
* ISTA ; TOPOI Excellence Cluster, DAI Berlin - anca.dan@topoi.org

I Cet article approfondit une recherche commencée en 2005, présentée au Colloque International « Orgamè/Argamum. À la recherche d'une colonie » (Bucarest-Tulcea-Jurilovca, 3-5 octobre 2005) et publiée préliminairement dans les Actes du Colloque..., réunis par M. Mănucu-AdameşTeanu, (Supplementum I de la collection Orgame/Argamum, 2006, p. 17I-205). Je remercie très chaleureusement Mme Mihaela Mănucu-Adameşteanu (Institut d'archéologie, Bucarest), M. Alexandru Avram (Université du Mans), Mme Renee Friedman (British Museum), M. Stephen Quirke (Petrie Museum), M. Pierre Dupont (CNRS Lyon) et les trois lecteurs anonymes désignés par $D H A$, pour leur aide. Que l'EA 2616 de l'Université de Reims, les bibliothèques d'égyptologie du Collège de France et de l'Université de Lille II, ainsi que tous ceux qui ont lu ces pages inédites (en particulier MM. Renaud Alexandre [Université de Paris IV-Sorbonne] et Claude Lemaire) trouvent ici le signe de ma gratitude.

2 Sur l'usage des termes « égyptien » et « faïence »cf. infra, note I4.

3 Un premier catalogue des objets « égyptiens » retrouvés en Méditerranée a été réalisé par J.D.S. PENDLebury, Aegyptiaca. A Catalogue of Egyptian Objects in the Aegean Area (foreword by H.R. Hall), Cambridge 1930. Il a été actualisé en 1994 dans la thèse, encore inédite, de N.J. SKon-Jedele, Aigyptiaka. A Catalogue of Egyptian and Egyptianizing Objects Excavated from Greek Archaeological Sites, ca. IIoo-525
} 
également les Grecs et les Barbares de la mer Noire . Les deux scarabées d'Orgamè que

BC, with Historical Commentary, University of Pennsylvania, qui reproduit (p. 2752-2852) les dessins des Aegyptiaca inventoriés (p. I-2693, 5138 objets) à partir d'une vaste bibliographie archéologique (p. 2694275I). En 2005, C. SAINT-Pierre a repris partiellement cette problématique dans une thèse d'histoire (Les offrandes orientales dans les sanctuaires du monde grec à l'époque archaïque, Université Paris I, inédite). Plusieurs parties du monde méditerranéen bénéficient maintenant d'un corpus des Aegyptiaca, grâce aux remarquables travaux de G. HöLв : l'Italie (Beziehungen der ägyptischen Kultur zu Altitalien, Leiden 1979), la Sardaigne (Ägyptisches Kulturgut im phönikischen und punischen Sardinien, Leiden 1986), Malte (Ägyptisches Kulturgut auf den Inseln Malta und Gozo in phönikischer und punischer Zeit, Wien 1989). Mentionnons aussi son étude méthodique consacrée à la Méditerranée orientale («Die Problematik der spätzeitlichen Aegyptiaca im östlichen Mittelmeerraum », dans Ägypten und der östliche Mittelmeerraum im I. Jahrtausend v. Chr. Akten des Interdisziplinären Symposions am Institut für Ägyptologie der Universität München 25.-27.10.1996, éds M. GöRG, G. HöLBL, Wiesbaden 2000, p. I19-162) ainsi qu'une conférence, restée inédite, sur la Méditerranée septentrionale, tenue lors du Workshop an der Ludwig-MaximiliansUniversität München, November 19g9. Malheureusement, des barrières politiques, économiques ou tout simplement linguistiques ont fait qu'aucun de ces catalogues n'inclut les découvertes du Pont-Euxin (à l'exception de la typologie d'A.F. GorTon, Egyptian and Egyptianizing Scarabs. A typology of steatite, faience and paste scarabs from Punic and other Mediterranean sites, Oxford 1996, qui se limite toutefois à la bibliographie de circulation occidentale, du début du $\mathrm{XX}^{\mathrm{c}}$ s., concernant les scarabées d'Olbia et de Bérézan).

4 Parmi les nombreux travaux sur les objets égyptiens découverts dans les villes et les territoires barbares autour de la mer Noire, nous renvoyons en premier lieu aux synthèses de B. TouraEfF, « Objets égyptiens et égyptisants trouvés dans la Russie méridionale », $R A$ I8, 1911, p. 20-35, еt « Скарабеи съ о. Березани », ИАK 40, I911, p. II8-I20; M. MATTHIEU, « Scarabs from South Russia », Ancient Egypt 3, 1926, p. 68-69 ; I. SNeguirefF, « Some Unpublished Egyptian Objects from Kertch, Olbia and Tiflis », Ancient Egypt 4, 1929, p. Іог-103, еt « Египет и юг СССР », Сборник работ студентоввъцвиженчев и студентов-иленов научньх кружков факультета истории, язька и материальной

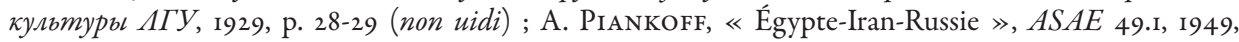
p. 5I-55; B.B. Ріотrowsкi, « Ägyptische Altertümer in norkaukasischen Gebiet », Сборник кружка по изучению Аревнего Востока при Государственном Эрмитаже, 1935, p. 35-49, et « Аревнеегипетские предметы, найденые на территории Советского Сосюза (Objets d'Égypte ancienne trouvés sur le territoire de l'Union Soviétique) », SovArch I, 1958, p. 20-27 ; М.А. Коростовцев, « Аревнеегипетские находки в СССР (Ancient Egyptian Objects found in the USSR) », VIMK 2, 1957, p. $72-86$; В.Б. ВинограАОв, « Египетские предметы из сарматского погребения близ города Грозного », SovArch 3, 1960, p. 309-310 ; А.К. КоровинА, « Фаиянсовые подвески из некрополей Тирамбы и Фанагории (Faience Pendants from the Necropolises of Tiramba and Phanagoria) », VDI II9.I, I972, p. IO4-II2 ; Е.М. ААЕКСеЕвА, « Предметы из египетского фаянса VI в. до н.э. - IV в. н.з. в Северном Причерноморье », KSIA 130, 1972, p. 3-11, еt Античные бусы Северного Причерноморья, Москва, 1975, p. 23-49, pl. 7-12 ; А.С. ОСтроверХов, « Фаянсовые и стекмянные бусы в Скифии и Сарматии (Faience and Glas Beads in Scythia and Sarmatia) », VDI 174.3, 1985, p. 92-109 ; Э.А. Сымонович, « Подражания амулетам из египетского фаянса в нижнем поднепровье (Imitations d'amulettes égyptiennes de faïence dans le bas Dniepr) », KSIA г45, 1976, p. 86-87 ; А.О. БОАьШАКОв \& Ю.И. ИАьнА, « Египетские скарабеи с острова Березань (Les scarabées égyptiens de l'île de Berezan') », VDI I86, 1988, p. 51-67 ; S. Hodjasch, « Skarabäen aus dem Territorium der Sowjetunion », dans so Years of Polish 
nous ajoutons aujourd'hui au dossier des Aegyptiaca apporteront peut-être quelques précisions sur l'étendue, dans l'espace et dans le temps, et sur les implications de ce phénomène historique qui reste encore largement à étudier.

Arrêtons-nous, tout d'abord, sur le contexte archéologique, bien enregistré et daté, dans lequel ont été trouvés les deux scarabées : il s'agit là d'une information dont on dispose rarement quand on étudie ce type d'objets'. Par conséquent, elle pourrait contribuer non seulement à la reconstitution de l'histoire orgaménienne et des relations, directes et indirectes, entre les régions les plus éloignées du monde ancien, mais aussi à l'établissement d'une typologie et d'une chronologie des scarabées égyptiens d'« époque tardive $»^{6}$.

Depuis 1991, Mihaela Mănucu-Adameşteanu organise des recherches annuelles dans la partie SE du promontoire Dolojman, zone correspondant, dans ses rapports annuels de fouilles, aux secteurs « Falaise Est » et, à partir de 1998, «Enceinte grecque ». Entre autres, elle veut répondre à une question posée il y a plusieurs années par Maria Coja, suite aux découvertes des années 1973-1976 : y avait-il, à l'époque grecque, une structure défensive qui entourait la ville ? Et, si l'on accepte l'existence d'une enceinte, quelles étaient ses dimensions, sa structure, son parcours, sa chronologie et son rapport avec les autres aménagements de la ville ${ }^{7}$ ?

Excavations in Egypt and the Near East. Acts of the Symposium at the Warsaw University 1986, Varsovie 1992, p. I22-I24; O. BERLEV \& S. Hodjash, Catalogue of the Monuments of Ancient Egypt from the Museums of the Russian Federation, Ukraine, Bielorussia, Caucasus, Middle Asia and the Baltic States, Göttingen 1998, p. 237 sq. (avec les remarques de E. Teeter, « (Review) Catalogue of the Monuments of Ancient Egypt from the Museums of the Russian Federation, Ukraine, Bielorussia, Caucasus, Middle Asia and the Baltic States », JNES 60.3, 2001, p. 193-194), Ancient Egyptian Scarabs. A catalogue of seals and scarabs from museums in Russia, Ukraine, the Caucasus and the Baltic States, Moscow 1999, p. 193-198, nos 1585-1656 ; Борисфен-Березань. Начало античной эпохи в Северном Причерноморье к го-летию археологических раскопок на острове Березань. Каталог выставки в государственном Эрмитаже 23 июня-гі сентября 20052, $\mathrm{n}^{\text {os } 196,200,201,203-205 .}$

5 Toutes les informations archéologiques (en grande partie encore inédites) ont été mises à ma disposition par Mme Mihaela Mănucu-Adameşteanu, auteur de ces fouilles.

6 On entend par là les objets datés après 664 av. J.-C. (date de l'avènement au trône de Psammétique I, premier représentant de la XXVI ${ }^{\mathrm{c}}$ dynastie) et avant la conquête macédonienne de $332 \mathrm{av}$. J.-C.

7 Dans son secteur II, Coja avait relevé un alignement de gros blocs façonnés en calcaire local, aujourd'hui encore visibles à l'extrémité Sud de la falaise orientale sur une longueur de 5-6 $\mathrm{m}$ et une hauteur de 0,400,60 m. Cf. M. Coja, Orgame/Argamum I. Cercetările dintre anii 1975-1985. Capitole dintr-o monografie arheologică inedită, éd. M. MĂNUCU-AdAMEşTEAnU, Bucureşti-Tulcea 2005, p. II, 22-23 (reprenant, en roumain, le texte « Les fortifications grecques dans les colonies de la côte ouest du Pont Euxin », paru dans La fortification dans l'histoire du monde grec. Actes du Colloque $\sigma_{14}$ du CNRS, Valbonne, décembre Ig82, éds P. Leriche, H. TréZiny, Paris 1986, p. 95-103 [I00]) et 6I-68. 
Plusieurs sondages ont été ouverts, entre 1998 et 2004 , à des intervalles réguliers sur la pente allant de la fortification d'époque justinienne au bord du lac Golovița ${ }^{8}$. On a mis ainsi en évidence un « réseau urbain d'habitations » ${ }^{9}$ organisé, au cours du $I^{e}$ s. av. J.- C., sur une terrasse limitée par une grande muraille identifiable, grâce à ses dimensions, comme « enceinte grecque $»^{10}$. Dès la première campagne, Mme MănucuAdameşteanu a repéré une belle maison grecque du dernier quart du $\mathrm{IV}^{\mathrm{e}} \mathrm{s}$. av. J.-C., située à l'intérieur des remparts et désignée, dans les rapports, comme « $\mathrm{LC}_{4} »$. C'est dans la « chambre 98 », particulièrement bien conservée, qu'elle a retrouvé, outre de nombreux fragments de céramique commune et fine partiellement jointifs, les deux scarabées que nous étudions ${ }^{11}$.

Le premier, $\mathrm{n}^{\circ}$ inv. ICEM $46773^{12}$ (cf.photo et dessin), découvert en 1998, provient d'une fosse qui descendait jusqu'à $125 \mathrm{~cm}$ de profondeur par rapport au niveau du sol moderne : les cendres qui la remplissaient indiquent sa fonction de « foyer domestique ». Dans cette même unité stratigraphique ont été retrouvés plusieurs tessons, parmi lesquels un timbre amphorique de provenance thasienne ( $\mathrm{n}^{\circ}$ inv. ICEM 44874). L'attribut de l'arc associé à des flèches et le texte $\Theta \alpha \sigma^{\prime} \omega \nu{ }^{\prime} \mathrm{H}^{\prime} \alpha \dot{k} \lambda \varepsilon \varepsilon \tau \odot \varsigma$, encore visibles sur cette anse, sont identiques à ceux de deux timbres d'Istros, datés, d'après le nom du magistrat, entre 329 et 317 av. J.- $\mathrm{C}^{13}$.

8 SIG (46 x 2 m, à 30 m Ouest du Secteur II de Maria Coja, en 1998-1999); cf. Cronica cercetărilor arheologice din România I999; SIG o0I (à $20 \mathrm{~m}$ Ouest de SIG, en 2000-200I); SIG 003 (20 x 2 m, à 20 m Ouest de SIG 00I, en 200I-2002), cf. Cronica...2001. Les sondages ont été complétés par cinq carrés décrits dans les mêmes rapports (résumés en français).

9 Cf. Cronica... 1997.

Io Épaisseur 2,3-2,6 m, plus de I8 $\mathrm{m}$ dégagés en longueur (sur l'aile méridionale), jusqu'à I,2 $\mathrm{m}$ de hauteur préservée. Cf. Cronica...2004; pour une description plus détaillée, $c f$. M. MĂnuCU-AdameşTEAnu, Orgamè/Argamum, ICEM-Tulcea, 200I, p. 44-46 (photos II-17), et « Orgame », dans D.V. Grammenos \& E.K. Petropoulos éds, Ancient Greek Colonies in the Black Sea Area, Thessaloniki 2003, p. 34I-388, surtout p. 347-350 (l'habitat classique) et 368-37I (l'inventaire mobile classique).

II Cf. Cronica... 1998 .

I2 Le sigle ICEM correspond à l'Institutul de Cercetări Eco-Muzeale Tulcea dont fait partie le Musée archéologique de Tulcea où sont conservées actuellement les trouvailles d'Orgamè.

I3 Cf. A. Avram, Histria VIII.I, Les timbres amphoriques I. Thasos, Bucureşti-Paris 1996, n² 244, avec l'analogie d'A.-M. Bon \& A. Bon, Les timbres amphoriques de Thasos. V. Grace collab., Paris 1957, n 7 I8b. D'après les statistiques d'A. Avram, 22 timbres avec le même nom de magistrat, répartis dans huit types, ont été découverts à Histria, cinq à Sinoé-Zmeica, in à Tomis, deux à Callatis, un à Odessos. 
Le deuxième scarabée, $\mathrm{n}^{\circ}$ inv. ICEM 46774 ( $c f$. photo et dessin), a été récupéré en 2000 , sur le sol en terre battue de la même maison, avec plusieurs fragments de céramiques fines (en particulier de canthares) datables également du dernier quart du $\mathrm{IV}^{\mathrm{e}} \mathrm{s}$. av. J.-C.

Examinons de plus près à la forme de ces scarabées : le premier mesure $1,25 \mathrm{~cm} \mathrm{~L}$ $\mathrm{x} 0,85 \mathrm{~cm} 1 \mathrm{x} 0,53 \mathrm{~cm} \mathrm{H}$ et a été modelé en «faïence égyptienne » ${ }^{14}$. Des analyses plus approfondies devraient être faites pour déterminer le type de glaçure et, éventuellement, la composition des faïences d'Orgamè. Ce type de travail n'étant pas encore envisageable, nous avons choisi de rester à la terminologie d'A. Lucas et de parler, de manière générale, de «typical egyptian faience ${ }^{\text {Is }}$. Le noyau, dans une pâte siliceuse

I4 Le terme « faïence », utilisé pour désigner « le matériau composite dont le corps, fait d'une pâte siliceuse, est recouvert d'une glaçure » ( $c f$. A. CAUbet \& G. Pierra-Bonnefois, Faïences. Faïences de l'Antiquité. De l'Égypte à l'Iran. Catalogue d'exposition, Musée du Louvre Io juin-I2 septembre 2005), originaire d'Égypte, était considéré comme impropre déjà par W.M.Fl. Petrie (qui lui préférait le terme «glaze »), à cause de la confusion possible avec la majolique, fabriquée à Faenza à partir de la fin du Moyen Âge. Pour le nom, la fabrication et la datation des faïences, voir maintenant P.T. NICHOLSON \& I. SHAw, Ancient Egyptian Materials and Technology, Cambridge 2000, p. 177-194 (chap. « Egyptian Faience ») qui a remplacé l'ouvrage fondamental d'A. Lucas, Ancient Egyptian Materials and Industries ${ }^{4}$, revised by J.R. HARRIs ( $\mathrm{I}^{\text {st }}$ edition 1926), London 1962, p. 155-178 (chap. « Glazed ware: glazed steatite, faience, faience variants, glazed quartz, glazed pottery, glazing methods and media »). Voir également P.T. Nicholson, Egyptian Faience and Glass, Shire 1993, et l'article homonyme dans l'OEAE (The Oxford Encyclopedia of Ancient Egypt, éd. D.B. Redford, Oxford 200i) ainsi que les excellents catalogues de F.D. Friedman (éd.), Gifts of the Nile. Ancient Egyptian Faience, London 1998, et A. Caubet \& G. Pierra-Bonnefois, Faïences.... Sur la composition chimique, il faut se reporter à l'étude d'A. KaCzmarczyK \& R.E.M. Hedges, Ancient Egyptian Faience : an analytical survey of Egyptian faience from predynastic to Roman times, avec un Appendix de P. VANDIER « Egyptian faience technology », Warminster I983, et aux essais d'archéologie expérimentale de J.V. Noble, «The Technique of Egyptian Faience », AJA 73.4, 1969, p. 435-439, et, plus récemment, de M.S. Tite et M. Bimson (e.g. «Egyptian Faience: an investigation of the methods of production », Archaeometry 25, 1983, p. 17-27, « Faience: an investigation of the microstructures associated with the different methods of glazing », Archaeometry 28, 1986, p. 69-78). Pour la faience « grecque », voir V. WeBB, Archaic Greek Faience. Miniature Scent Bottles and Related Objects from East Greece, 650-50o BC, Warminster 1978 .

I5 Ancient Egyptian Materials..., p. 157. Les six variantes qu'il proposait pour ce type principal ont été d'ailleurs récupérées dans les typologies ultérieures et apparentées aux trois techniques fondamentales de fabrication : efflorescence, application, cimentation. Cf. A. Kaczmarczy \& R.E.M. Hedges, Ancient Egyptian Faience..., p. I85 sq. et A26 sq. ; P.T. Nicholson \& I. SHAw, Ancient Egyptian Materials..., p. 177, 186 sq. 


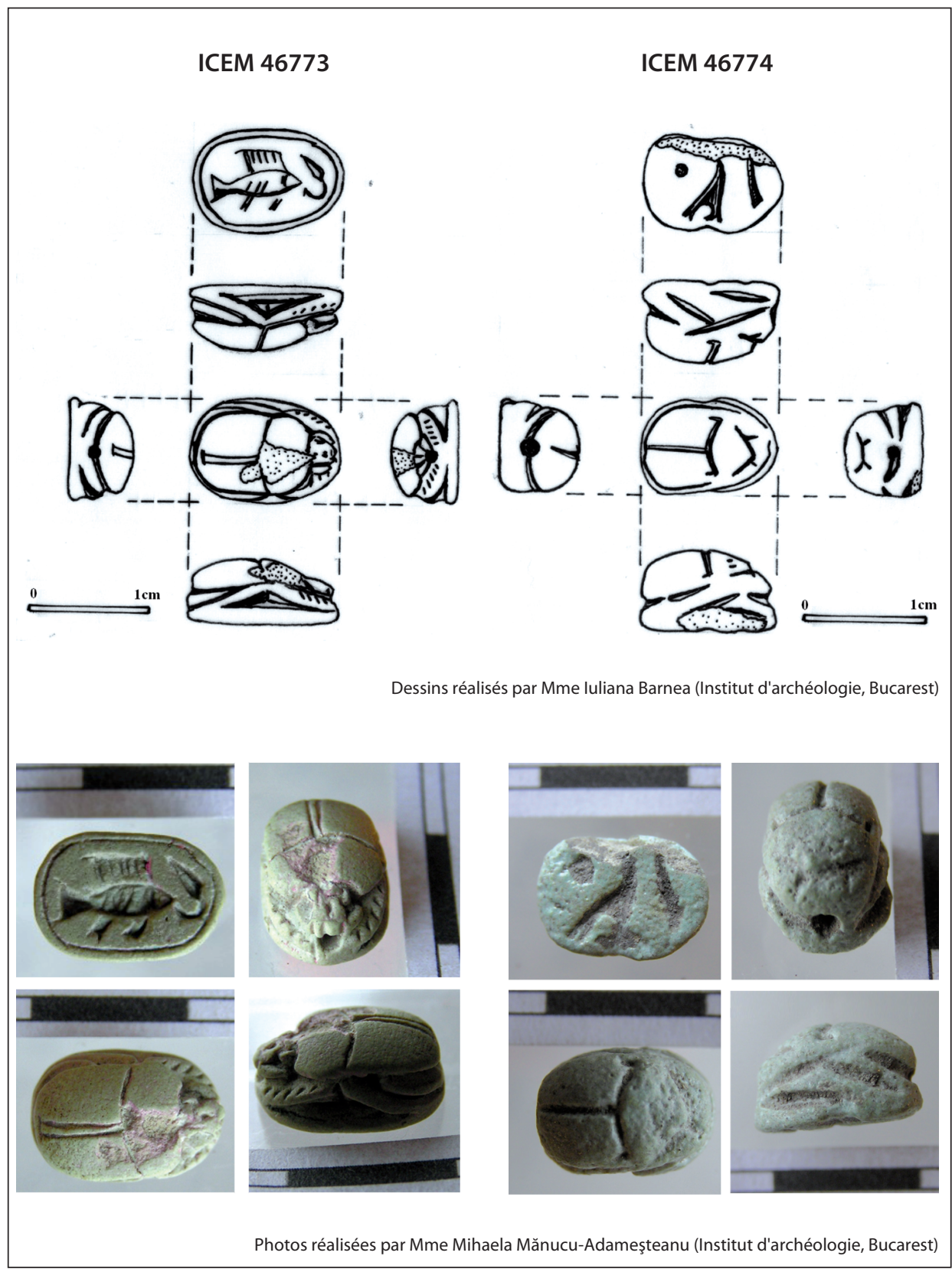


friable, peu rugueuse, de couleur verdâtre ${ }^{16}$, est recouvert uniformément d'une glaçure alcaline vert-jaune printemps, assez fine ${ }^{17}$.

La qualité supérieure du produit est confirmée par les détails de la représentation mi-naturaliste mi-conventionnelle du Scarabaeus Sacer : le clipeus, délimité par les deux yeux ou les deux antennes, présentait, antérieurement à l'éclatement local de la glaçure, trois palpes s'avançant devant la tête. Le thorax (pronotum), de forme ovale, est séparé des élytres par un trait simple, légèrement arrondi. Un double segment figure schématiquement la suture des deux élytres, s'arrêtant, par un bref trait perpendiculaire, avant l'extrémité postérieure du corps. Les trois paires de pattes hautes, en saillie par rapport au corps, se fondent dans une base de 0,10-0,12 cm d'épaisseur ; seuls les membres antérieurs sont décorés par cinq stries visibles sur la face principale et sur les côtés de chacun. L'insecte est percé, dans le sens de la longueur, d'un conduit de section circulaire, destiné à l'insertion dans la monture métallique d'une bague ou dans un collier ${ }^{18}$. Sur la partie plate, le décor, encerclé d'un trait fin, a été adroitement incisé (ou peut-être, vu l'aspect particulièrement régulier, estampé), avant la cuisson, en position horizontale, à partir de la tête du scarabée, dans le sens habituel de la lecture égyptienne : c'est un indice intéressant pour déterminer la fonction principale de l'objet, destiné en conséquence plus à une lecture directe (amulette) qu'à une lecture à travers son empreinte (sceau) ${ }^{19}$. L'entaille représente un poisson : sur le corps fusiforme (sa longueur comprend deux fois et demi sa largeur), on distingue la représentation de l'œil et de la tête pointue, séparée du corps par un trait vertical. Plusieurs hachures, sché-

I6 Ce qui est « normal » pour une production utilisant « iron-rich sands of Egypt» qui « usually yield grey or brown cores $\gg, c f$. A. Kaczmarczyk \& R.E.M. Hedges, Ancient Egyptian Faience..., p. 194, qui précise (p. 188) : «Very little statistical data is available regarding the frequency with which some effort was made to either use quartz or select and purify high-silica sands. [...] No more than $20 \%$ of the faience bodies could be termed as white, while the majority were distinctly brown or grey, a clear indication of the presence of oxides or iron ». Sur la faïence d'《époque tardive », cf. ibidem, p. 265 sq.

I7 Sur la couleur verte, de bon augure dans les croyances orientales, $c f$. J. BOARDMAN, « Colour Questions », Jewellery Studies 5, 1992, p. 29-31 ; voir également les théories sur le symbolisme de la faïence, liée à la renaissance, dernièrement chez P.T. Nicholson \& I. Shaw, Ancient Egyptian Materials... ; A.A. MARAVELIA, « Ancient Egyptian Inscribed Faience Objects from the Benaki Museum in Athens I », JNES 61.2, 2002, p. 8I-I09 ; A. Caubet \& G. Pierra-Bonnefois, Fä̈ences....

I8 Sur les témoignages iconographiques attestant le port des scarabées (à Chypre), voir E. LAGARCE dans G. Clerc \& V. Karageorghis \& E. Lagarce \& J. Leclant, Fouilles de Kition II. Objets égyptiens et égyptisants: scarabées, amulettes et figurines en pâte de verre et en faïence, vase plastique en fä̈ence. Sites I et II, 1959-75, Nicosia, 1976, p. 167 sq. Voir également, dans le même volume, p. 21 sq., les principes de description des scarabées que nous avons essayé de suivre.

19 C'est d'ailleurs généralement le cas des scarabées découverts en contexte grec. $C f$., entre autres, A.F. GorTon, Egyptian and Egyptianizing Scarabs.... 
matisant peut-être les écailles et leurs différences chromatiques, reproduisent symétriquement les sept arrêts obliques de la nageoire dorsale ; celle-ci est représentée par un parallélogramme qui longe l'épine dorsale entre les opercules et le pédoncule caudal ; la nageoire anale est raide, concave à l'extrémité, orientée obliquement vers la queue ; la nageoire pectorale suit la direction de l'anale, tout en étant plus souple et suggérant ainsi le mouvement de la nage ; la nageoire caudale est parfaitement triangulaire, les deux épines qui forment ses extrémités dépassant sensiblement ce cadre géométrisé.

La forme du corps et des nageoires nous permet de reconnaître ici une représentation de Tilapia Nilotica ${ }^{20}$. De sa bouche sort une tige doucement arrondie, terminée vers le haut par un renflement effilé en pointe qui remplit, avec un souci de symétrie, l'espace resté vide; il s'agit de la reproduction très populaire du bouton du lotus bleu, 9, Nymphaea Caerulea (Savigny I798) 21.

Cette description rapproche notre objet, dans la première tentative de classification des scarabées faite par P.E. Newberry (Egyptian Antiquities. Scarabs. An Introduction to the Study of Egyptian Seals and Signet Rings, London, 1906), des figurations « naturalistes » de la XII ${ }^{e}$ (I994-178I av. J.-C., fig. 60) et de la XVIII ${ }^{e}$ dynastie (I540I295 av. J.-C.). La forme détaillée et soignée des scarabées de ces époques a été privilégiée pendant la XXVI ${ }^{\mathrm{e}}$ dynastie (664-525 av. J.-C.), qui manifestait, au cours de la «Renaissance saïte », une volonté de retour aux thèmes plastiques du passé ${ }^{22}$. Nous pourrions, en effet, faire remonter l'ascendance morphologique de notre exemplaire aux scarabées du type IV de G.T. Martin (datés de la XII ${ }^{e}$ dynastie) et à leurs sous-types

20 Pour l'identification des poissons représentés par les Égyptiens, voir P. MONTET, « Les poissons employés dans l'écriture hiéroglyphique », BIFAO II, 1914, p. 39-48 (surtout 39-40, Tilapia dans les hiéroglyphes); Cl. GAILLARD, Recherches sur les poissons représentés dans quelques tombeaux égyptiens de l'Ancien Empire, V. Loret, Ch. Kuentz collab., Le Caire 1923, p. 85-88 (Tilapia dans la peinture) ; P. Chimits, «Tilapia in Ancient Egypt », FAO Fishery Bulletin 10.4, 1957, p. 21I-215 (non vidi); M. DAmBACH \& I. WALlert, « Das Tilapia-Motiv in der altägyptischen Kunst », Chronique d'Égypte 4I, 1966, p. 273-294. Voir aussi, pour d'autres poissons, L. KeIMER, « Quelques représentations rares de poissons égyptiens remontant à l'époque pharaonique », BIF 29 (session 1946-7), 1948, p. 263-274, et F. DAumas, « Quelques remarques sur les représentations de pêche à la ligne sous l'ancien empire », BIFAO 62, 1964, p. 67-85, etc. 2I M.-A. Beauverie, « Description illustrée des végétaux antiques du Musée égyptien du Louvre », $B I F A O$ 35, 1935, p. II5-15I, surtout I 46 sq. (avec une explication des caractéristiques botaniques qui différencient le lotus blanc du lotus bleu) et BENSON-HARER, «Lotus » dans OEAE (200I). Pour le symbole, voir, avec bibliographie, E. BRUNNER-TRAUT, « Lotos » (1979), dans L Ä (= Lexikon der Ägyptologie, dir. W. Helck, E. Otto ; éds W. Helck, W. Westendorf, Wiesbaden 1975-1986).

22 Cf. déjà G. Fraser, A Catalogue of the Scarabs Belonging to George Fraser, London 1900, VIII ; H.R. Hall, Scarabs, London 1929, p. II sq. 
(de la XVIII ${ }^{e}$ dynastie $)^{23}$ : on y retrouve déjà un clipeus dentelé, un seul trait qui divise le prothorax des élytres et deux traits pour séparer les élytres. En l'absence d'une classification des scarabées d'《époque tardive » ou d'《époque grecque » (ou, du moins, de «type naucratite $\left.{ }^{24}\right)$, nous proposons d'intégrer la découverte orgaménienne au type XXVIII A (groupe VI, « Naukratis Factory and Forerunners ») de Gorton ${ }^{25}$ : ont été regroupés ici des scarabées retrouvés à Naucratis, à Carthage, en Espagne, en Italie méridionale, en Grèce continentale (Perachora, Sounion), à Rhodes (Camiros, Lindos), à Chios ainsi que huit des 26 scarabées inventoriés dans les colonies septentrionales de la mer Noire (d'après Touraïef, Matthieu et Алексеева) ${ }^{26}$. La date de fabrication de ce groupe correspondrait à la période d'activité de l'atelier fouillé par Petrie (594-570 av. J.-C., d'après les noms des pharaons Psammétique II et Apries marqués sur les scarabées et la céramique grecque qui leur était associée) ${ }^{27}$.

23 Cf. G.T. Martin, Egyptian Administrative and Private-name Seals Principally of the Middle Kingdom and Second Intermediate Period, Oxford 1971, p. 4, I5 I. Sur toutes ces typologies, $c f$. E. Hornung \& E. Staehelin, Skarabäen und andere Siegelamulette aus Basler Sammlungen, Mainz 1976, p. 32-33.

24 Promise néanmoins par A.F. GorTon, Egyptian and Egyptianizing Scarabs... Pour le deuxième millénaire, une telle typologie a été mise au point grâce aux travaux de Tufnell, Ward et Dever $(c f$. W.A. WARD, Studies on Scarab Seals I. Pre-12 ${ }^{\text {th }}$ Dynasty Scarab Amulets, Warminster 1978 ; O. Tufnell, Studies on Scarab Seals II (I and 2). Scarab Seals and Their Contribution to History in the Early Second Millennium BC. Contributions by G.T. Martin, W.A. Ward, Warminster 1984 ; W.A. WARD \& W.G. Dever, Studies on Scarab Seals III. Scarab Typology and Archaeological Context. An Essay on Middle Bronze Age Chronology, Texas 1994). Voir aussi B. JAEger, Essai de classification et datation des scarabées Menkhéperrê, Göttingen 1982.

25 A.F. Gorton, Egyptian and Egyptianizing Scarabs..., p. 93-107, I41, I44, I78-180. Le type est défini (p. 93) par la matière (« a rough composition of creamy colour usually covered with a green [...] glaze »), la forme («scarabs usually have a prothorax outlined [...] by a curved line [...]. The elytra are divided and a $V$-shaped incision is often placed on either side. The legs are always deeply carved. Their average size is I.I $x 0.8$ $x 0.6 \mathrm{~cm} »)$ ), le sujet («Group A includes scarabs and scaraboids with representational themes ») et le style ( The main characteristic [...] of this type is the use of a cast technique for the devices. The style is homogenous. [...] The hieroglyphs (type XXVIIIB) are not very clearly presented [...]. Representations and inscriptions occupy the whole field »). Néanmoins, J. Boardman avertit le lecteur, dans la préface, que beaucoup de scarabées trouvés sur des sites non-puniques n'entrent pas dans cette typologie.

26 A.F. GorTon, Egyptian and Egyptianizing Scarabs..., p. 174, pour les scarabées des colonies pontiques: 《 all datable to the period of the Greek expansion in this area in the late $7^{\text {th }}$ and $\sigma^{\text {th }}$ cent. 》

27 Sur cet atelier, voir W.M.Fl. Petrie, Naucratis I (I884-I885), London I886, p. 5, 6, I4, 22, et la description sommaire des fouilles, p. 36-39. En plus des nombreux scarabées, il avait trouvé ici 678 moules différents de ceux trouvés habituellement sur les sites égyptiens. Son successeur, E.A. GARDNER (Naucratis II, Appendix F.L. GRIfFith, London I888), se contente d'inclure dans sa publication une planche de scarabées dessinée par Petrie, sans autre mention. Le dernier à fouiller sur le site de l'atelier, à un moment où les infiltrations gênaient déjà les travaux, a été D.G. HogARTH (avec C.C. EDGAR, C. GUTCH, « Excavations at Naukratis », BSA 5, I898-1899, p. 22-97, et, avec H.L. Lorimer et C. EDGAR, « Naukratis », 
Regardons de plus près le décor dessiné sur la partie plate : le Tilapia Nilotica (Linnaeus 1758 ) est couramment appelé bolti (d'après la désignation courante des arabophones), chromis (suivant Cuvier et d'autres savants du XIX ${ }^{\mathrm{e}} \mathrm{s}$.) ou carpe du Nil ${ }^{28}$, (in.t) en égyptien ${ }^{29}$. Ce poisson a été, pendant toute l'histoire, l'un des plus familiers aux Égyptiens et, par conséquent, les plus figurés dans leur art. C'est un

1903, JHS 25, 1905, p. 105-136) : il insiste sur l'appartenance de cette partie méridionale de la ville aux Égyptiens et sur l'origine non-grecque (c'est-à-dire égyptienne ou phénicienne) des artisans. H. PRINZ (Funde aus Naukratis, Beiträge zur Archäologie und Wirtschaftgeschichte des VII. und VI. Jahrhunderts $v$. Chr., 1908 [réimpr. 1963], p. 99-108) donnera une première étude (unique jusqu'au travail de Gorton) sur la distribution égéenne des scarabées « naucratites 》 (voir, p. I06, ses références aux aryballes et aux scarabées de Bérézan). Comme les recherches archéologiques contemporaines (dirigées par W.D.E. CoulsoN et A. Leonard Jr., voir Cities of the Delta, part I. Naukratis. Preliminary Report on the 1977-78 and 1980 Seasons, Malibu 1981, et Ancient Naukratis vol II. The Survey at Naukratis and Environs, Part I. The Survey at Naukratis, Oxford 1996) n'ont plus accès à la ville, les historiens de l'art tendent aujourd'hui à nier l'existence de la fabrique de Petrie, considérant le nombre de moules trouvés insignifiant et les découvertes de scarabées à Rhodes plus importantes ( $c f$. A. Caubet \& G. Pierra-Bonnefois, Faïences..., p. I5, et C. Saint-Pierre, Les offrandes ..., p. 235 etc., ayant comme point de départ les recherches de V. WebB, Archaic Greek Faience..., p. 3 sq., qui ne met pourtant jamais en doute l'atelier naucratite). Les principales synthèses sur la faïence (P.T. Nicholson \& I. Shaw, Ancient Egyptian Materials..., p. i84 sq.), celles qui s'intéressent à l'économie de Naucratis (J. Boardman, The Greeks Overseas. Their early colonies and trade $^{4}$, London 1999 [I $\mathrm{I}^{\mathrm{st}}$ ed. 1964, réimpr. 2000] ; A. MöLler, Naukratis. Trade in ancient Greece, Oxford 2000, p. I13-II5, 152-154, reprenant la classification de Gorton) acceptent la thèse de Petrie, à notre sens irréfutable, sur la présence d'une fabrique de faïences à Naucratis. Pour un résumé critique des hypothèses chronologiques (VII ${ }^{\mathrm{e}}-\mathrm{VI}^{\mathrm{e}}$ ou $\mathrm{VI}^{\mathrm{e}} \mathrm{s}$. av. J.-C.) et ethniques (les artisans étaient-ils égyptiens, grecs ou phéniciens ?), cf. dernièrement P. James, « Naukratis Revisited », Hyperboreus, 9.2, 2003, p. 235-264, surtout p. 252 sq., qui développe une idée suggérée par Hogarth : l'atelier, phénicien, n'aurait fonctionné qu'avant l'établissement des Grecs à Naucratis au temps d'Amasis.

28 Pour les noms modernes, scientifiques et communs, cf. G.A. Boulenger, Zoology of Egypt: The Fishes of the Nile, London 1907, p. 513, 523 sq. ; I. Gamer-WALlert, Fische und Fischkulte im Alten Ägypten, Wiesbaden 1970, p. 13-14; FIGIS 2004 - Tilapia (sur www.fao.org, 2004).

29 Cf. A. ERman \& H. Grapow, Wörterbuch der aegyptischen Sprache im Auftrage der deutschen Akademien, Leipzig 1926-1953 (réimpr. Berlin 1971), s.u. « ein Nilfisch (« bulti »; Tilapia Nilotica) »; le signe a une valeur figurative (déterminative) aussi bien que phonétique. Pour tous les noms identifiables pour ce poisson et leurs occurrences, $c f$. I. GAMER-WALlERt, Fische..., p. 24-27, et « Tilapia », dans L $\ddot{A}$ (19751978) : on retient le nom $w 3 \underline{d}$ (cité infra, dans le «Livre des morts ») qui compte, parmi ses sens, celui d'《 être vert, jeune », cf. A. ERMAN \& H. GRAPOW, Wörterbuch..., s.u.). Tilapia pourrait correspondre, en

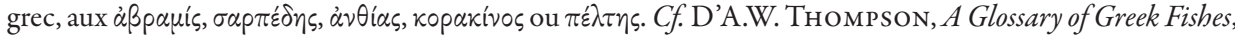
Oxford 1947, s.u., et la présentation des sources grecques chez Th. HopfNer, « Der Tierkult der alten Ägypter nach den griechisch-römischen Berichten und den wichtigeren Denkmälern », Denkschriften der Kaiserlichen Akademie der Wissenschaften in Wien. Philosophisch-Historische Klasse 57.2, 1913, p. 150 sq. 
représentant de la famille des Cichlides, ordre des Perciformes ${ }^{30}$. Il était répandu originairement dans les eaux douces du continent africain, du lac de Tibériade et du bassin du Jourdain jusqu'au Nil (avec une éventuelle préférence pour le Delta), ainsi que dans le lac Tchad, la Volta et le fleuve Sénégal. Sa taille (jusqu'à 50-60 cm de longueur pour un mâle adulte), son abondance et le goût agréable de sa chair en font, depuis toujours, «one of the commonest luxuries of the people $»^{31}$. En effet, comme les études modernes l'ont montré, le fameux tabou des Égyptiens ${ }^{32}$ relatif soit à l'impuretée ${ }^{33}$ soit à la sacralité $^{34}$ des poissons ne concernait que le roi (pendant sa vie ainsi qu'après sa mort), les prêtres et certaines villes pendant certains jours de l'année ${ }^{35}$. Pour le peuple, le poisson,

30 Pour les débats des taxonomistes sur les divisions en genres et sous-genres des Tilapiinés, $c f$. P. Kestemont \& J.C. Micha \& U. Falter, Les méthodes de production d'alevins de Tilapia Nilotica, Projet de l'Organisation des Nations Unies pour l'alimentation et l'agriculture (disponible dans les Archives des documents de la FAO, www.fao.org), 1989, chap. I, « Biologie de la Tilapia Nilotica ». Voir également l'étude la plus récente de C.E. Lim \& C.D. WeBster éds, Tilapia: biology, culture, and nutrition, NY (USA) 2006.

3I Cf. G.A. Boulenger, Zoology of Egypt..., p. 513-528 (p. 528).

32 Sur le tabou (et le symbole du poisson dans sa graphie hiéroglyphique), voir P. MonTET, « Le fruit défendu. Cours du Collège de France, décembre 1949 », Kêmi II, 1950, p. 85-116 ; pour l'absence des poissons dans les tombes pharaoniques, voir P. LACAU, «Suppressions et modifications de signes dans les textes funéraires », Z $\ddot{A} S$ 5I, 1913, p. I-64. Il s'agit d'ailleurs, cf. S. LAGERCRANTZ, « Forbidden fish », Orientalia Suecana 2, 1953, p. 2-8, d'un interdit commun aux peuples hamites.

33 Comme le raconte Plut., Isis et Osiris, $358 \mathrm{~b}$ (éd. W. Sieveking, 1935, Teubner), ce sont trois poissons, le lépidote, le phagre et l'oxyrhynque, qui ont dévoré le sexe d'Osiris, lorsque son frère Seth avait répandu les parties déchirées de son corps sur le Nil. Néanmoins, on connaît des villes qui honoraient ces poissons par leur nom, tel qu'il nous est connu en grec (Oxyrhynchos, aujourd'hui El-Behnasa, près de Béni Mazar ; Lepidoton Polis, aujourd'hui Nag el-Mashayikh, près d'Abydos). De plus, les archéologues ont découvert un cimetière d'oxyrhynques momifiés à El-Omari, des exemplaires en bronze dans la ville sainte d'Osiris, Abydos etc. Tilapia paraît être particulièrement honoré à Bubastis et, associé à Hathor, à Denderah.

34 Comme le mentionne le calendrier $n^{\circ} 86637$ du Caire (publié en volume par A.M. BAKIR, en 1966), au $22^{\mathrm{c}}$ jour de la saison de l'inondation, le Soleil a craché les corps des dieux, qu'il avait dévorés, sous forme de poissons et leurs esprits $(\mathrm{Ba})$ sous forme d'oiseaux. $C f$. I. GAMER-WALlERT, Fische..., p. 76-85 ; A. Belluccio, « Les poissons célestes », dans Proceedings of the VII ${ }^{\text {th }}$ International Congress of Egyptologists, Cambridge 3-9 september 1995, éd. C.J. EYrE, Leuven 1998, p. 129-142.

35 Comme le $28^{\mathrm{c}}$ jour du $\mathrm{IV}^{\mathrm{c}}$ mois d'Akhit, où l'on ne mange pas de poisson, car ceux qui résident à Mendès avaient été transformés en in.t. Cf. P. MonteT, « Le fruit défendu... », p. 98 ; Chr. DesrochesNoblecourT, « Poissons, tabous et transformations du mort. Nouvelles considérations sur les pèlerinages aux villes saintes », Kêmi 13, 1954, p. 33-42 (surtout p. 38) ; L.-A. ChristophE, « Le ravitaillement en poissons des artisans de la nécropole thébaine à la fin du règne de Ramses III », BIFAO 65, 1967, p. 177-199, surtout p. 98 ; D. MeEKs, « Le nom du dauphin et le poisson de Mendès », REg 25, 1973, p. 209-216. 
éventuellement séché ou salé, était un aliment de base, les Égyptiens pouvant même passer, aux yeux des lecteurs d'Hérodote, pour des Ichtyophages ${ }^{36}$.

C'est peut-être l'aspect chromatique de ce poisson (du jaune au brun olivâtre, avec des taches foncées) et les nuances rouge-pourpre de ses nageoires ${ }^{37}$ ainsi que son apparition, les matins du solstice d'été, dans les eaux moins profondes du fleuve ${ }^{38}$, qui ont déterminé son association avec le Soleil : en effet, Rê n'est pas seulement le créateur des poissons mais, durant son itinéraire nocturne, il devient lui-même poisson, combattant dans l'eau contre le monstre des ténèbres. Étant donnée la comparaison/ identification du circuit de la barque solaire pendant la nuit et du parcours de la barque d'Osiris après la mort, les représentations des Tilapia dans les tombes ne sont pas inhabituelles $^{39}$ : les scènes de pêche sont ainsi liées au souci du mort de sortir victorieux de sa transformation en poisson, juste avant sa renaissance ${ }^{40}$.

36 Cf. Hdt. 2.92 : «Quelques-uns de ces Égyptiens vivent uniquement de poissons; quand ils les ont pris et vidés, ils les font sécher au soleil et les consomment ensuite une fois secs » (trad. Ph.-E. LEGRAND). Le fait qu'Hérodote associe dans le même chapitre les Lotophages aux Ichtyophages pourrait être significatif. Sur l'identification de Tilapia dans Hdt. 2.93, 99, voir entre autres M. DAMBACH \& I. WALLERT, « Das Tilapia-Motiv... », p. 278-28o. Pour des exemples précis révélés par l'archéologie, on peut penser à l'alimentation avec des poissons des ouvriers de Déir el-Médineh au début de la $\mathrm{XX}^{\mathrm{e}}$ dynastie ; voir L.-A. Christophe, « Le ravitaillement... ». Pour la nourriture des armées de Séthi I, $c f$. J.H. Breasted, Ancient Records from Egypt. Historical Documents from the Earliest times to the Persian Conquest, vol. 3 (XIX ${ }^{\text {th }}$ Dynasty), I906-1907, no 207.

37 Cf. e.g. D.J. Brewer \& R.F. Friedman, Fish and Fishing in Ancient Egypt, The Natural History of Egypt II, Warminster, 1989. Sur les tentatives des artistes de rendre les couleurs du poisson, voir L. KeImeR, « Notes de lecture (suite) », BIFAO 56, 1957, p. 97-I20.

38 Cf. infra, sur la reproduction des Tilapia et la note 48.

39 Cf. Cl. Gaillard, Recherches sur les poissons... Sur les offrandes de poissons, voir T. Handoussa, « Fish Offering in the Old Kingdom », MDAIK 44, 1988, p. I05-109. Pour une synthèse sur le rôle des poissons (dont Tilapia) dans le contexte funéraire, $c f$. I. GAMER-WALlert, Fische..., p. I20 sq., et D. SAhrhage, Fischfang und Fischkult im alten Ägypten, Mainz 1998, p. 29 sq.

40 Cf. Chr. Desroches-Noblecourt, « Poissons... »; A. Belluccio, « Les poissons... »; sur la transformation du défunt en poisson dans le chapitre 153 du Livre des morts, voir Ph. GERMOND \& J. Livet, Bestiaire égyptien, Paris, 2001 p. 48-49. 
Dans un deuxième temps, à l'époque de la composition du « Livre des morts ${ }^{41}$, Tilapia est le guide et le protecteur de la barque solaire, celui qui aide Rê dans sa lutte contre le monstre Apophis ${ }^{42}$ :

Belle est la barque de Rê en cette belle heure du jour ! Tu te lèves bellement, ô Soleil ; (sept fois). L'avant de la barque est tourné vers l'Occident, pour le départ. Le fils de Nout porte ses armes ; il a tué la tortue, il a repoussé l'oryx, il a chassé le serpent. Le poisson-ouadj jubile, le poisson-abdjou est en fête, les dieux dans le ciel sont en acclamation [...].

Louanges à toi $[. .$.$] ; ta mère Nout t'a mis au monde d'elle-même [...]. Tu es monté au ciel, tu$ as traversé les eaux célestes [...]. Tu as vu le poisson-inet dans sa forme (véritable) sur la rivière de turquoise ; tu as vu le poisson-abdjou quand il vient à se manifester ; le serpent mauvais a été renversé comme il (l'a) annoncé, et j'ai planté mes deux couteaux (dans) ses vertèbres; alors Rê fut dans un vent favorable [...] et tous les dieux étaient dans la jubilation [...] (chap. XV, traduction P. Barguet) ${ }^{43}$.

Cette fonction protectrice pour le Soleil et pour le mort dans le combat contre les ennemis de l'ordre détermine, à l'époque tardive ${ }^{44}$, l'identification du Tilapia avec Sekhmet, « la Puissante » déesse à tête de lionne, ennemie d'Apophis ${ }^{45}$. Finalement, le Tilapia est utilisé directement dans la magie, comme instrument prophylactique pour les vivants, et devient symbole occulte ${ }^{46}$.

4I C'est-à-dire à partir de la XVIII ${ }^{e}$ dynastie, mais remontant, pour certains textes, jusqu'à la Première période intermédiaire. Une introduction à cette problématique est faite par P. Barguet, Le Livre des morts des anciens Égyptiens. Introduction, traduction, commentaire, Paris 1967.

42 Cf. E. Hornung \& A. Badawy, s.u. dans LÄ (1986); S. Sauneron, s.u. dans G. Posener (collab. S. Sauneron et J. Yoyotte), Dictionnaire de la civilisation égyptienne, Fernand Hazar, Paris 1959 ; Cl. Traunecker, s.u. dans DA (= Dictionnaire de l'Antiquité, éd. J. Leclant, Paris 2005).

43 Pour l'interprétation du passage, voir J. Assmann, Egyptian Solar Religion in the New Kingdom: Re, Amun and the crisis of polytheism (trad. A. Alcock), London - New York 1995, p. 34, 38-57 (qui ajoute aussi des passages du Livre du jour) et, brièvement, E.A. WALlis Budge, From Fetish to God in Ancient Egypt, New York 1988, p. 98.

44 D'ailleurs, cette image a été préservée jusqu'à l'époque copte et dans le Christianisme, $c f$. F.J. DöLGER,

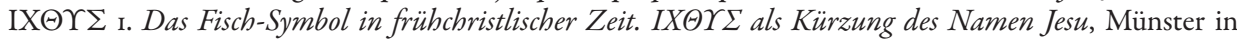
Westsh 1928 ; voir aussi le commentaire de quelques inscriptions chrétiennes par G.R.H. WRIGHT, « On Fishes and Men. Fish symbols in ancient religion », JPR I, 1987, p. 30-43; pour une représentation de Tilapia avec une fleur dans sa bouche à l'époque copte, cf. M. DAмвACH \& I. Wallert, « Das TilapiaMotiv... », p. 293 (fig. II, d'après Mond \& MYers, Temples of Armant, London 1940, fig. 25).

45 Pour la phrase « Je suis la déesse-int et devant moi tremblent les dieux qui sont dans la prairie marécageuse (ainsi que ?) ceux qui sont dans le lac-mint $\gg$ dans une formule de protection du roi contre les dangers de l'année, voir J.-Cl. Goyon, «Un phylactère tardif : le Papyrus 3233 a et b du Musée du Louvre », BIFAO 77, 1977, p. 45-54 (surtout p. 53-54, avec bibliographie).

46 Y. Koenig, « Un revenant inconvenant? (Papyrus Deir el-Médineh 37) », BIFAO 79, 1979, p. IO3-II9 (surtout p. 107-108). 
Néanmoins, ce qui a le plus frappé les habitants du Nil jusqu'à l'époque moderne et ce qui pourrait justifier la popularité de ce poisson, c'est son mode de reproduction (qui est celui des Cichlidae en général). On sait aujourd'hui que :

Lorsque les conditions abiotiques deviennent favorables, les adultes migrent vers la zone littorale peu profonde et les mâles se rassemblent en arène de reproduction sur une zone en pente faible à substrat meuble, sablonneux ou argileux où ils délimitent chacun leur petit territoire et creusent un nid en forme d'assiette creuse. [...] En cas d'arrêt au-dessus d'un nid et après une parade nuptiale de synchronisation sexuelle, la femelle dépose un lot d'ovules que le mâle féconde immédiatement et que la femelle reprend en bouche pour les incuber. [...] L'éclosion des œufs a lieu dans la bouche, 4 à 5 jours après fécondation. Une fois leur vésicule vitelline résorbée ( \pm Io jours après éclosion), les alevins capables de nager sont encore gardés par la femelle pendant plusieurs jours. Toutefois, ils restent à proximité de leur mère et, au moindre danger, se réfugient dans sa cavité buccale ${ }^{47}$.

Les anciens Égyptiens avaient remarqué ce phénomène d' « incubation buccale ${ }^{48}$; ils l'expliquaient, peut-être, comme les pêcheurs nilotiques du XIX ${ }^{\mathrm{e}}$ siècle :

But it is universally known to the fishermen of Egypt, who, Mr. Loat says, firmly believe that the eggs get into the mouth by a reversed mode of partution. ${ }^{49}$

L'animal qui dévore sa membrane vitelline pour venir au monde et qui, une fois adulte, met au monde de sa propre bouche sa progéniture, ne saurait être que l'emblème $\mathrm{du}$ « renouveau de la vie ${ }^{50}$. Il fait nécessairement penser au dieu Atoum qui a créé le monde en répandant sa semence de sa bouche, et, d'une manière plus générale, à l'idéal égyptien de monogenèse et d'autogenèse qui a consacré le scarabée lui-même ${ }^{\text {II }}$.

47 P. Kestemont \& J.C. Micha \& U. Falter, Les Méthodes..., chap. I.6 « Biologie de la reproduction $\gg$. Sur la différentiation moderne des quatre genres des Tilapiinés, d'après l'incubation buccale et la « garde parentale », ibidem, chap. I.I.

48 Comme argument des connaissances égyptiennes sur la fabrication d'un nid, $c f$. peut-être les représentations sur les bols de plusieurs poissons disposés en cercle : M. DAMBACH \& I. WALLERT, « Das TilapiaMotiv... » (fig. 7-8, avec analogies); D. SAHrhage, Fischfang..., I23 sq. (fig. I8).

49 Le premier savant moderne à avoir remarqué que les femelles étaient responsable de ce phénomène, a été G.A. Boulenger, Zoology..., p. 527-528 : « The nursing habit which this species has in common with so many members of the family Cichlidae was first pointed out by me in a female from Lake Galilee, forming part of Canon Tristram's collection. [...]The eggs are oval, the greater diameter being about 3 millimetres, and one female carries from 200 to 800 . 》

so Cf. R. Hri, « Le poisson-scarabée comme amulette : deux documents inédits », Genava N.S. 3I, 1983, p. $5-8$.

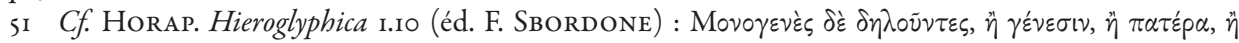

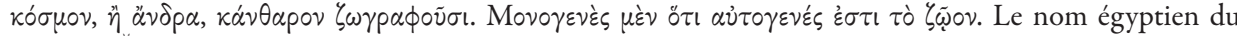

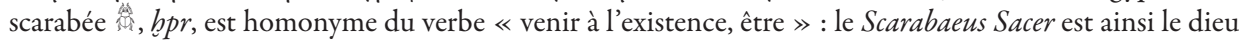
Khepri, le Démiurge / Soleil levant, celui qui naît de lui-même, tout en existant ( $c f$. « Le livre d'Apophis » : « Le seigneur de l'Univers dit : quand je me fus manifesté à l'existence, l'existence exista », apud 
Une dernière remarque ichtyologique enrichit une fois de plus le symbolisme du Tilapia : le lotus qui sort de sa bouche ne saurait guère nous étonner, car nous avons affaire à un poisson qui se nourrit principalement du phytoplancton. Néanmoins, la Nymphaea caerulea représentée ici indique plus que la nourriture de Tilapia ou qu'une marque du Nil : c'était le lotus bleu qui flottait sur les eaux au début du monde et c'est de sa fleur épanouie, parfumée tous les matins, que s'échappa le Soleil. Horus/Harpocrate, dieu de la lumière, sort de l'eau assis sur une fleur entrouverte de lotus de même que Nefertoum, fils de Sekhmet ${ }^{{ }^{2}}$. Le lotus, en bois ou en terre cuite, était d'ailleurs à lui seul une amulette ${ }^{53}$ : d'autant plus forte devait être, pour l'Égyptien connaisseur, l'association d'un Tilapia tenant un lotus dans sa bouche, avec le scarabée, image par excellence de la vie et de la régénération ${ }^{54}$.

Vu le nombre des liens possibles entre Tilapia et la croyance des Égyptiens en la renaissance, la multitude et la diversité de ses représentations ne sauraient guère

Y. CAMBefort, Le scarabée et les dieux. Essai sur la signification symbolique et mythique des coléoptères, Paris 1994). Comme l'attestent Plut., De Iside et Osiride 355A, 380F-38IA Stephanus et Horap., Hieroglyphica I.IO, I2, les anciens Égyptiens considéraient que l'insecte, toujours mâle, déposait ses œufs dans une boule de terre qu'il roulait en la poussant de ses pattes postérieures, comme le Soleil qui, s'avançant de l'Orient à l'Occident, allait dans la direction opposée du mouvement de la voûte céleste. Sur ce sujet, cf., après les titres de la bibliographie de G.T. MarTin, Scarabs, Cylinders and Other Ancient Egyptian Seals. A checklist of publications, Warminster 1985 (avec une mention spéciale pour F. DE SALvia, « Un ruolo apotropaico dello scarabeo egizio nel contesto culturale greco-arcaico di Pithekoussai (Ischia) 》 dans Hommages à M.J. Vermaseren. Recueil d'études offert par les auteurs de la Série EPRO..., éds. M.B. DE Boer et T.A. Edridge, Leiden 1978, p. 1003-106I) ; R.S. BiAnchi, s.u. dans LÄ (1984) et dans OEAE (200I) ; D. Ben-Tor, The Scarab : A Reflection of Ancient Egypt, Jerusalem 1989 ; W.A. WARD, « Beetles in Stone : the Egyptian scarab », BiblArch 57.4, Dec. 1994, p. 182-202 ; C. ANDrEws, Amulets of Ancient Egypt, British Museum 1994, p. 50-53 ; K.M. CoOney \& J. Tyrrell, Scarabs in the Los Angeles County Mouseum of Art. Part I Distributed propaganda or intimate protection?, Stanford University, 2005 (disponible aussi sur www.PalArch.nl). Pour une brève information sur les cosmogonies égyptiennes, voir la notice de D. Valbelle dans $D A$.

52 Voir S. Sauneron \& J. Yoyotte, La naissance du monde selon l'Égypte ancienne, Paris I959, p. 54-58; S. Morenz \& J. Schubert, Der Gott auf der Blume, Ascona 1954. Rappelons également le chapitre 8I $\mathrm{du}$ «Livre des morts » : « je suis ce pur lotus qui sort portant le Lumineux, celui qui est attaché au nez de Rê ; je suis descendu le chercher pour Horus. Je suis le pur, qui sort de la prairie marécageuse » (trad. P. BARGUET).

53 E.g. W.M.Fl. Petrie, Buttons..., p. I2, 14, pl. II-IV.

$54 C f$., entre autres, B. BRUYÈRE, « L'enseigne de Khabekhnet », BIFAO 28, 1929, p. 4I-48, surtout p. 45 ; Z.B.W. KRÖNIG, « Ägyptische Fayenceschalen des Neuen Reiches », MDAIK 5, 1934, I44-166. Pour un bref inventaire des animaux (dont Tilapia) représentés régulièrement sur des scarabées, voir A. GrENFELL, «Les divinités et les animaux figurés sur les scarabées; sur les scaraboïdes ; les plaques ; les chatons et les amulettes », Rendiconti della R. Accademia dei Lincei I7, Ser. $5^{\circ}$, fasc. I. (seduta del 16 febbraio I908), p. 135-155. 
nous surprendre. Des « palettes » découvertes dans les tombes pré-dynastiques, des peintures funéraires de l'Ancien Empire, des vases décorés et surtout des bols en faïence turquoise consacrés, au cours du Moyen et du Nouvel Empire, dans les tombes ou dans les sanctuaires d'Hathor ${ }^{55}$, jusqu'aux scarabées et scaraboïdes fabriqués pendant plus d'un millénaire, l'histoire plastique des poissons Tilapia est pratiquement impossible à inventorier ${ }^{56}$. Des nombreux exemples d'amulettes qui apparaissent dans les monographies de sites égyptiens et dans les catalogues des musées ${ }^{57}$, les plus significatives pour notre sujet nous paraissent les découvertes de W.M.Fl. Petrie à

55 Sur Tilapia et Hathor, voir Chr. Desroches-Noblecourt, « 'Un lac de turquoise'. Godets à onguents et destinées d'outre-tombe dans l'Égypte ancienne », MonPiot 47, 1953, p. I-34, et « Poissons... »; E. STAEHELIN, «Zur Hathorsymbolik in der ägyptischen Kleinkunst », Z $\ddot{A} S$ I05, 1978, p. 76-84, surtout p. 8I-82 ; A.A. Maravelia, Faience Objects of the Ancient Egyptian Collection of the National Archaeological Museum in Athens I, Göttingen 2002 (p. 6I-72 + plates), et surtout la synthèse critique de G. PINCH, Votive Offerings to Hathor, Oxford 1993, p. 287-288.

56 Voir cependant les synthèses de M. Dambach \& I. Wallert, « Das Tilapia-Motiv... »; I. GamerWALlERT, Fische..., la meilleure étude aujourd'hui sur la question ; D.J. Brewer \& R.F. Friedman, Fish... ; D. SAHrhage, Fischfang..., avec bibliographie ; S. Hodjasch, « Zum Motiv Tilapia auf altägyptischen Kunstwerken in der Museen den Sowjetunion », Göttingen Miszellen, 1991, p. 39-48. Les plus anciennes représentations semblent être les palettes d'Hiéroconpolis (J.E. QUiBELL, Hierakonpolis I, London 1900 , pl. XXI, fig. I6, pl. XXII, ph. I8). Pour Tilapia avec lotus, un inventaire des vases, avec explication, a été donné par Z.B.W. KRÖNIG, « Ägyptische... » (surtout p. I57 sq.).

57 Nombreux sont les scarabées et les scaraboïdes décorés d'un ou de deux Tilapia en Égypte, surtout pendant et après la XVIII ${ }^{c}$ dynastie : e.g. P.E. New Berry, Egyptian Antiquities..., pl. XXV, n ${ }^{\text {os }} 3 \mathrm{II}^{-32}$ (XVIII $^{c}$ dynastie) ; W.M.Fl. Pet RIE, Tell el Amarna, London 1894, p. I49; Idem, Hyksos and Israelite Cities, London 1906, pl. XI, n ${ }^{\circ} 22$; Idem, Gizeh and Rifeh, London 1907, pl. XXIII, n' 21 ; Idem, Memphis I, London 1909 , pl. XXXIV, ${ }^{\circ}$ Ior ; Idem, Amulets. Illustrated by the Egyptian Collection in University College London, I914, $\mathrm{n}^{\circ} 257 \mathrm{a}-\mathrm{f}$; Idem, Buttons..., n $\mathrm{n}^{\text {os }}$ 923-928, I4I3 ; R. ENGELBACH \& B. GunN, Harageh, London 1923, pl. $2 \mathrm{I}$ $\mathrm{n}^{\circ} 194$; A. Rowe, A Catalogue of Egyptian Scarabs, Scaraboids, Seals and Amulets in the Palestine Archaeological Museum, Le Caire 1936, n 597 ; J. Bourriau \& A. Millard, « The Excavation of Sawâma in I9I4 by G.A. WAINWRIGHT and T. WhitTEMORE », JEA 57, I97I, p. 28-57, nºs 30, 32, 47 ; E. HornUnG \& E. Staehelin, Skarabäen..., Bi7, B45, B82 (et p. ilo-iII) ; F.S. Matouk, Corpus du scarabée égyptien, t. II Analyse thématique, Beyrouth 1977, dessins 826-831, photo II88 ; R. GIVEON, Egyptian Scarabs from Western Asia from the Collections of the British Museum, Göttingen 1985, Gezer 59 10495I et Amrit I3, 48245 ; Idem, Scarabs from Recent Excavations in Israel, éds D. WARburton, Ch. UeHLinger, Göttingen 1988, n ${ }_{11} 6$; S. HodJASCH, «Zum Motiv Tilapia... », n ns 5 -II, certains repris dans Eadem, Ancient Egyptian Scarabs..., $\mathrm{n}^{\text {os }}$ I170-1175 (avec d'autres analogies); G. ClERC, « Aegyptiaca » dans Études chypriotes XIII. La Nécropole d'Amathonte. Tombes IIO-385, éds V. Karageorghis, O. Picard, Chr. Tytgat, Nicosie 1991, T 240/73; 283/203; 321/106 (avec de nombreuses analogies et interprétation p. 49-50); Ch. Herrmann, Die ägyptischen Amulette der Sammlungen BIBEL+ORIENT der Universität Freiburg Schweiz. Anthropomorphe Gestalten und Tiere, Göttingen 2003, n ${ }^{\text {os }} 932-934$ (avec parallèles) ; E. TEETER, Scarabs, Scaraboids, Seals, and Seal Impressions from Medinet Habu. With Post-Pharaonic Stam Seals and Seal impressions by T.G. Wilfong. Based on the fields notes of U. Hölscher and R. Anthes, Chicago 2003, 
Naucratis. Malheureusement, nous ne disposons ni d'image ni de description pour la partie convexe et le profil des scarabées, ce qui nous interdit toute conclusion d'apparenté assurée avec notre scarabée. De plus, le contexte archéologique des trouvailles, dont les dessins faits par Petrie ont été publiés dans les deux volumes Naucratis, n'a pas été noté : une grande partie des scarabées a été d'ailleurs apportée par les ouvriers et l'auteur omet de mentionner lesquels ont été découverts dans la fabrique (et donc dans un contexte pour lequel il propose une datation) et lesquels ont été récupérés dans la ville (datés donc éventuellement d'après 570 av. J.-C.). Néanmoins, le scarabée $\mathrm{n}^{\circ} 5$, pl. XVIII de Naucratis II ainsi que le scaraboïde $\mathrm{n}^{\circ} 157$, pl. XXXVIII de Naucratis I prouvent l'existence du motif «Tilapia avec lotus bleu » dans le répertoire des scarabées présents dans l'emporion, probablement dans la première moitié du VI ${ }^{\mathrm{e}}$ siècle av. J.-C. Notre scarabée aurait donc pu être produit à Naucratis, dans l'atelier fouillé par Petrie ou dans un autre, détruit à jamais ; mais, étant donné le succès de son motif décoratif et l'impossibilité de l'insérer dans une typologie plus précise du point de vue de l'origine et de la chronologie, il pourrait également venir d'un tout autre atelier du Delta et avoir été exporté vers l'Égée par l'intermédiaire de Naucratis (si cela a eu lieu avant 525 av. J.-C.) ou même par un autre établissement grec (si ce fut après la conquête perse de l'Égypte) ${ }^{58}$.

Quant au sens que les Grecs en général et les habitants pontiques d'Orgamè en particulier auraient pu donner à un scarabée en faïence portant sur sa base un poisson avec un bouton de fleur, toutes les spéculations demeurent possibles ${ }^{59}$. Aussi conviendrait-il d'admettre qu'après l'adoption du scarabée comme forme principale d'amulette dans le monde grec et syro-phénicien, à partir du VI ${ }^{e}$ s. av. J.-C. ${ }^{60}$, tout objet de ce genre devait être interprété comme talisman (à fonction apotropaïque). Essayons toutefois d'être plus précis : si notre scarabée est le seul, à ce jour, à porter ce décor parmi les amulettes égyptiennes découvertes autour de la mer Noire, les vases en faïence rhodienne, reproduisant le même Tilapia, n'étaient pas inconnus aux Grecs

$\mathrm{n}^{\text {os }}$ 135, 137 ; F. MAGNARINI, Catalogo ragionato di una collezione di Scarabei-Sigillo Egizi, BAR International Series I24I, 2004, 09.10 etc.

$58 C f$. infra, pour la discussion de la situation à l'époque perse.

59 Sur la perception et la fonction des scarabées égyptiens dans le monde grec en général, voir la belle analyse de F. DE SAlvia, « Un ruolo... ».

60 Voir à ce sujet J. BoARdman, Archaic Greek Gems. Schools and artists in the sixth and early fifth centuries BC, London 1968, p. 13-17, et Greek Gems and Finger Rings. Early Bronze Age to Late Classical, London 1970, p. 139 sq. 
pontiques ou, du moins, à ceux de Bérézan ${ }^{61}$. D’une manière plus générale, l'image d'un poisson aurait pu être associée à l'idée de fécondité ou de vitalité et ne serait ainsi pas surprenante sur une amulette portée par une femme ou par un enfant.

Le deuxième scarabée d'Orgamè se différencie du précédent à plusieurs égards : du point de vue des dimensions $(1,15 \mathrm{~cm} \mathrm{~L} \mathrm{x} \mathrm{0,7} \mathrm{cm} \mathrm{l} \mathrm{x} \mathrm{0,67} \mathrm{cm} \mathrm{H})$, il est moins grand mais plus haut. La faïence dans laquelle il a été moulé, appartenant toujours à la catégorie « égyptienne proprement dite » de Lucas, est de qualité inférieure : le noyau, visible grâce à une longue cassure au niveau des « pattes » droites et de l'extrémité de la base, est constitué d'une pâte assez compacte mais rugueuse, de couleur blanche-grisâtre. Comme le cœur des faïences est généralement teinté, en raison des impuretés présentes dans le sable, une couleur blanche peut être considérée commel'indice d'un processus volontaire de purifier la matière première. Le but de l'artisan aurait été, dans ce cas, d'obtenir, après l'application de la glaçure, une couleur mieux contrôlée, ce qui s'accorderait, éventuellement, à une époque «tardive ${ }^{62}$ ou à un contexte non-égyptien ${ }^{63}$. La glaçure, de couleur verte-turquoise, foncée, sans brillance, a été appliquée en couche fine, homogène, laissant encore transparaître de nombreuses irrégularités à la surface de l'insecte. De plus, les détails du moule sont réduits à l'essentiel : la tête et le thorax ne sont suggérés que par un trait en forme de $\mathrm{V}$, schématisant leur articulation. Les deux points situés près de l'angle du $\mathrm{V}$, vers l'extérieur, sont les seules marques de l'emplacement d'un clipeus. Les élytres, délimités entre eux par un seul trait, sont séparés de ce qui devrait être le thorax par une ligne légèrement arrondie : sa longueur correspond à l'ouverture

6I Cf. S.L. Solovyov, Ancient Berezan. The architecture, history and culture of the first Greek colony in the Northern Black Sea, Leiden 1999, p. 86 fig. 77, découvert dans la nécropole datée de la deuxième moitié du $\mathrm{VI}^{\mathrm{c}}$ et de la première moitié du $\mathrm{V}^{\mathrm{c}} \mathrm{s}$. av. J.-C. Ce vase est à ajouter à l'inventaire de V. WeBB, Archaic Greek Faience ..., p. I34 sq., n ${ }^{\text {os }}$ 941-949, pl. XXI-XXII.

62 Cf. A. Kaczmarczy \& \& R.E.M. Hedges, Ancient Egyptian Faience, p. I88 sq.

63 Cf. T.J. Dunbabin éd., Perachora. The Sanctuaries of Hera Akraia and Limenia, Excavations BSAA 1930-1933. Vol. II, Pottery, Ivory, Scarabs and Other Objects from the Votive Deposit of Hera Limenia, Oxford 1962, p. 461 sq., à propos des scarabées de Perachora: « The core is extremely fine in texture whereas Egyptian composition is coarse. [...] It <the glaze > seems to have been applied as a skin, while on Egyptian composition objects the glaze is more closely incorporated in the core [...]. The green glaze is of a different quality also ». Pourtant, les analyses spectrographiques n'ont pas montré de différences par rapport aux faïnces trouvées en Égypte (p. 467 sq.). Chr. Blinkenberg, Lindos I. Fouilles de l'Acropole 19o2-IgI4. Les petits objets, Berlin I93I, p. 37 I sq., précise que le noyau des scarabées de Lindos est d' « un blanc pur », même s'il les considère comme issus d'une main égyptienne de Naucratis. 
du V qui lui fait face. Chacune de ses extrémités est marquée par un minuscule trait orienté vers la partie postérieure du corps, pour donner une idée de la forme par ailleurs non représentée des élytres. Les vues de profil montrent une tentative de marquer les contours du corps et les pattes par quelques incisions asymétriques et complètement aberrantes. Comme le premier, ce deuxième scarabée d'Orgamè est percé en longueur par un conduit circulaire pour une monture. Sur la partie plate, on distingue un décor incisé horizontalement, lisible aussi bien sur l'objet (comme sur une amulette) que sur son empreinte (en tant que sceau). De droite à gauche, comme l'indique l'orientation des hiéroglyphes, on identifie : la partie inférieure linéaire, légèrement recourbée, d'une plume (probablement d'autruche et de Mâat, ) ; le segment supérieur ayant disparu lors de la cassure, il nous est impossible de préciser si la plume était réduite à un trait ou si elle était arrondie. Le deuxième signe représente un oiseau d'une allure imposante : ses deux pattes confondues forment un T renversé, grossièrement tracé, probablement à cause des aspérités de la céramique et d'un défaut de la base non-aplatie du scarabée. Le corps est assez fin, élancé et les ailes sont distinguées des plumes caudales par un petit espace ; le cou est long et se terminait, sans autre démarcation, par une tête dont il nous est impossible aujourd'hui de préciser la forme et l'orientation. On pourrait donc hésiter entre l'identification d'un faucon $\mathbb{N}$ (vu la sveltesse du dessin, même caricaturé, et la fréquence du motif sur les scarabées) ou d'une chouette La troisième figure (soit la première dans une lecture de gauche à droite) représente, avec quelques imperfections, un cercle entièrement creusé, où l'on reconnaît facilement le disque $\odot$.

Il est bien difficile de trouver une place pour ce scarabée dans les typologies existantes : le principal obstacle est, encore une fois, au-delà du manque de catalogues pour les scarabées égyptiens ou égyptisants de l'époque qui nous concerne, l'absence des dessins et des descriptions des dos et des profils dans les publications actuellement disponibles ${ }^{64}$. Ce corps représenté très schématiquement, sur la base duquel on a gravé, d'une manière plutôt maladroite, trois hiéroglyphes qui ne paraissent pas, à première lecture, cohérents, pourrait être apparenté au type XXII (e.g. n 140) de Gorton : découverts en Asie Mineure, en Grèce et en Italie et datés du VIII ${ }^{\mathrm{e}}$ ou du VII ${ }^{\mathrm{e}}$ siècle av. J.-C., ces scarabées sont similaires et probablement dérivés d'un même type que ceux qui seront fabriqués, presque un siècle plus tard, à Naucratis (type XXVIII de Gorton). La simplicité de la forme (un seul trait sépare le thorax des élytres et les élytres entre

64 E.g. W.M.Fl. Petrie, Naucratis..., qui ne donne que le dessin du décor ainsi qu'une sélection de in impressions réalisées avec les moules découverts, dont cinq uniquement sont de scarabées (pl. XXXVIII ; cf. aussi la photo de J. BoArdman, The Greeks..., p. I29 fig. I5I). A.F. GorTON, Egyptian and Egyptianizing Scarabs..., ne publie, pour son type XXVIII, aucun dos de scarabée. 
eux) et le répertoire réduit des symboles incisés ou cachetés sur la base (combinaisons de trois hiéroglyphes « aux pouvoirs magiques ») caractérisent la production massive de ces faïences, peut-être dans des ateliers situés en terre égéenne (éventuellement à Rhodes ${ }^{65}$.

Cependant, à en juger d'après les hiéroglyphes inscrits sur la base, c'est encore à Naucratis que nous trouvons un parallèle pour le scarabée orgaménien : le scarabée $\mathrm{n}^{\circ} 21 \mathrm{~b}$ (de Naucratis II, pl. XVIII), mesurant, d'après le dessin à l'échelle 1:1, 0,11 cm L x $0,7 \mathrm{~cm} \mathrm{l}$, présente, en position verticale, le regroupement des hiéroglyphes même combinaison de signes, dans la même position verticale, se retrouve sur le scarabée $\mathrm{n}^{\circ} 712$ publié, en photographie, par Petrie en 1925 (pl. XII, dimensions 0,11 cm L x $0,8 \mathrm{~cm} \mathrm{l}$ ), sans aucune information sur la provenance et la datation, mais avec une lecture (p. 21) : «On 712 are named Ra Horus and Shu or Maot ${ }^{66}$. Néanmoins, si sur notre scarabée, à la place du faucon on reconnaît le signe de la chouette c'est dans le Pont-Euxin même, et plus précisément à Olbia, que l'on trouve une analogie pour la découverte d'Orgamè. Il s'agit d'un scarabée découvert par B. Pharmakovsky dans une tombe lors de sa campagne de 1911. De la même sépulture archaïque proviennent quatre autres scarabées, avec les mêmes caractéristiques morphologiques que celui qui nous intéresse :

They are all made of a very soft paste which turns easily to powder. [...]. The form of the scarabs is very simple: the elytra are outlined, and a line also divides the elytra from the thorax; the head, clypeus and eyes, as well as legs, are roughly marked [...] They are all about $0.4 \mathrm{inch}$ long, and all are made of light-blue paste. [...] In the same tomb with these scarabs three pendants were found; [...] The pendants in form of lions were found at Naucratis [...]. In the tombs, together with our scarabs (as can be traced from diaries of Prof. Pharmakovsky), were found black-figured vases, Rhodian, Miletian and Naucratite ware, porcelain and glass beads, which of course fully correspond with the fact of the Naucratite origin of our scarabs ${ }^{67}$.

65 Cf. A.F. Gorton, Egyptian and Egyptianizing Scarabs..., p. 72, qui intègre cette production au premier type de faïence rhodienne de V. WebB, Archaic Greek Faience .... Néanmoins, Webb ne prend pas en discussion les scarabées. P. Dupont \& V. Nazarov, « Amphores levantines en mer Noire », H $_{3}$ истории античного общества 8, 2003, p. I42-I48, surtout p. I44 = I47 n. 8, attirent l'attention sur ces productions : « Le rôle traditionnellement attribué à Rhodes dans la fabrication et la diffusion d'objets en faïence de style égyptisant [...] reste encore en grande partie à démontrer. Comme dans le cas du style des Chèvres Sauvage ou de celui de Fikellura, la fréquence des trouvailles pourrait bien faire illusion ».

66 On pourrait éventuellement proposer encore W.M.Fl. Petrie, Naucratis..., pl. XXXVII, p. 53-54.

67 Sur ces fouilles, voir la bibliographie apud S.D. Kryzhytskyy \& V.V. Krapivina \& N.A. Lejpunskaja \& V.V. Nazarov, « Olbia - Berezan », dans D.V. Grammenos \& E.K. Petropoulos éds, Ancient Colonies..., p. 389-505, surtout p. 392-393. 
Après cette première publication de Matthieu ${ }^{68}$, le scarabée d'Olbia a été inséré par Алексеева dans le type 50, variante I, de son catalogue de 1975 (p. 4I-42 et pl. 9 : IIБ) : des 26 scarabées qu'elle attribue à cette catégorie, 24 proviennent d'Olbia. La plupart d'entre eux trouvent des analogies parmi les scarabées de Naucratis. La chercheuse russe propose aussi une datation du groupe, entre la deuxième moitié du VI et la première moitié du $\mathrm{V}^{\mathrm{e}} \mathrm{s}$. av. J.-C. Ses dessins (pl.9) seront réimprimés pour l'illustration de l'article de J. Bouzek ${ }^{69}$, ayant comme objet les objets « orientaux », apportés, peutêtre, par des commerçants phéniciens dans le Pont-Euxin.

En dehors de la mer Noire, c'est encore Naucratis qui nous offre une analogie possible, avec le $\mathrm{n}^{\circ}$ io2 $\mathrm{pl}$. XXXVII dans la monographie homonyme de Petrie $^{70}$. Mentionnons aussi les scarabées de la collection du Caire ${ }^{0 s} 36854,36984$ et peutêtre 37016, publiés par Newberry dans le Catalogue général des antiquités égyptiennes du Musée du Caire, $n^{o s}$ 3600I-37521. Scarab-shaped Seals, de 1907. On retrouve clairement la même succession de hiéroglyphes ${ }^{\odot}$, toujours en position horizontale, sur le scarabée 98I du corpus de Matouk. Malheureusement, on ne dispose d'aucune information sur la provenance de ce scarabée « de collection », que son propriétaire date de la «Basse Époque ». Par conséquent, plus intéressants s'avèrent être les scarabées découverts dans les nécropoles de Carthage, portant sur leurs bases des inscriptions similaires à celle du scarabée olbien : il s'agit, tout d'abord, de la série d'au moins cinq scarabées publiés par Vercoutter ( $\mathrm{n}^{\text {os }}$ 293-297), en provenance de la nécropole Douïmès et conservés, au moment de la publication, au musée Lavigerie. Ces scarabées mesurent tous I-I,I cm L, $0,8-0,85 \mathrm{~cm} \mathrm{l}$ et $0,55-0,65 \mathrm{~cm} \mathrm{H}$. Ils sont confectionnés en pâte dure ou friable, de couleur blanche ou verte, et sont datés du VII ${ }^{e} s$. au VI ${ }^{e} s$. av. J.-C., d'après la chronologie « générale » de cette nécropole d'incinération ${ }^{71}$. Ajoutons aussi le $\mathrm{n}^{\circ}{ }^{172}$,

$68 C f$. «Scarabs... », $\mathrm{n}^{\circ} 4{ }^{\circ}$. Sur le parallèle, apparemment faux, établi par Matthieu entre son scarabée $\mathrm{n}^{\circ} 4$ et le $\mathrm{n}^{\circ} 84$, pl. XXXVII de Naucratis $I 3^{\odot}$, voir notre discussion sur l'interprétation de l'inscription infra. Un rapprochement similaire a été fait par A. MORET, « Catalogues des scarabées et intailles au Musée Alaoui à Tunis », $B A C$, I911, p. I60-167 $\left(\mathrm{n}^{\circ} 9 \text { lu comme } \beta\right\}^{\circ}{ }^{\odot}$, alors qu'une lecture 11 nous paraît également possible). Sur la céramique « naucratite », cf. J. BOARDMAN \& C.E. VAPHOpoulou-Richardson éds, Chios. A conference at the Homereion in Chios 1984, Oxford 1986, p. 215 sq. ; R.M. Cook \& P. Dupont, East Greek Pottery, London-New York 1998.

69 «Les Phéniciens en mer Noire? 》 dans Civilisation grecque et cultures antiquespériphériques. Hommage à Petre Alexandrescu à son $70^{\circ}$ anniversaire, éds A. Avram, M. BABEş, Bucureşti 2000, p. 134-137, surtout p. 137, sans autre référence dans le texte.

70 Cependant, vu la qualité du dessin, nous ne pouvons guère nous prononcer sur la forme et la valeur du hiéroglyphe que l'on attendrait pour ?.

7I J. Vercoutter, Les objets égyptiens et égyptisants du mobilier funéraire carthaginois, Paris I945, p. 2I-22. La nécropole avait été fouillée à la fin du XIX'c s. par le R.P. Delattre. 
du VI ${ }^{e}$ s. av. J.-C., découvert dans la tombe 216 de Dermech ${ }^{72}$ et déjà publié par Moret $\left(n^{\circ} 9\right)$. Ces datations concordent avec la période d'activité de l'atelier de Naucratis où Vercoutter propose de situer éventuellement l'origine de ces scarabées appartenant à son type VII ${ }^{73}$. C'est également l'avis de Gorton (p. I03 sq.) qui reprend dans son type XVIII B (scarabées avec inscriptions hiéroglyphiques imprimées, en provenance de Naucratis) les nos. 293-297 de Vercoutter ${ }^{74}$.

On pourrait donc parler d'un consensus sur la datation (fin VII $/$ début $\mathrm{VI}^{\mathrm{e}}-\mathrm{V}^{\mathrm{e}} \mathrm{s}$. av. J.-C.) et sur l'origine (Delta occidental ou Naucratis, avec distribution ultérieure vers la Méditerranée occidentale - Carthage, la Méditerranée orientale - Égypte, et la mer Noire - Olbia et désormais Orgamè) de ces scarabées en faïnce, de qualité médiocre, de dimensions similaires, portant une inscription de type $\odot$ ou $\odot$.

C'est d'ailleurs cette inscription qui suscite des interprétations divergentes. Aucun dictionnaire et aucune grammaire d'égyptien hiéroglyphique ne fait référence à un mot ou à une phrase dont la graphie pourrait être rapprochée de ces groupes de trois hiéroglyphes. Beaucoup de chercheurs ont considéré qu'il n'y avait pas de sens derrière ces signes, qu'ils étaient là pour un public non-égyptien, amateur d'exotisme. D’autres, comme Vercoutter et, après lui, Gorton, ont pensé à une abréviation : si à la même époque, sur des scarabées du même type « naucratite », on pouvait, par exemple, lire comme raccourci pour Psammétique ${ }^{\text {er } 75}$, pourquoi ne pas voir, sur les $\mathrm{n}^{\text {os }}$ 293-297 de Vercoutter, l'inscription I qu'on pourrait lire comme $\mathbb{R}$ (avec l'omission de $i b), W h m$-[ib]-Re, nom de Néchao II, qui n'est pas présent sur d'autres scarabées $?^{76}$ Enfin, d'autres, comme Matthieu, ont voulu rapprocher notre inscription d'une autre, beaucoup plus fréquente ${ }^{77}$ et, d'une certaine manière, intelligible :

72 J. Vercoutter, Les objets égyptiens..., p. 22-26 pour la datation de la nécropole, au $\mathrm{VI}^{\mathrm{c}}-\mathrm{V}^{\mathrm{c}} \mathrm{s}$. av. J.-C.

73 Le type VII, «Scarabées à technique spéciale » de Vercoutter (Les objets égyptiens..., p. 162 sq., $\mathrm{n}^{\text {os }} 269$ sq.), est caractérisé par la constance des dimensions ( $\mathrm{cm} \mathrm{L} \mathrm{x} \mathrm{o,8} \mathrm{cm} \mathrm{l} \mathrm{x} \mathrm{0,6} \mathrm{cm} \mathrm{H),} \mathrm{de} \mathrm{la} \mathrm{technique}$ (matière moulée) et des inscriptions.

74 Mentionnons également l'exemplaire en serpentine de J. ŚLIwa, Egyptian Scarabs and Magical Gems from the Collection of C. Schmidt-Ciaziniski, Nakładem Uniwersytetu Jagiellońskiego 1989, n 22 pl. VIII, qui dispose les hiéroglyphes (auxquels on pourrait éventuellement ajouter $\bullet$ ) en position verticale, rappelant les scarabées avec cités plus haut.

75 Cf. W.M.Fl. Petrie, Scarabs and Cylinders with Names. Illustrated by the Egyptian Collection in University College London, 1917, p. 32 (pl. LV); J. VerCoutTer, Les Objets égyptiens..., p. 57, 77.

76 Cf. J. Vercoutter, Les objets égyptiens..., p. 77. L'absence du nom de ce pharaon est d'ailleurs un argument important pour ceux qui veulent rabaisser la date de l'atelier fouillé par Petrie à Naucratis (cf. P. James, « Naukratis... »).

77 E.g. P.E. Newberry, Catalogue général..., n ${ }^{\text {os }} 36314,36319,37058,37059$, 3734I-37343 (pl. VIII) ; W.M.Fl. Petrie, Naucratis..., pl. XXXVII 83-84, 86-88, pl. XXXVIII 175. Voir les listes d'occurrences 


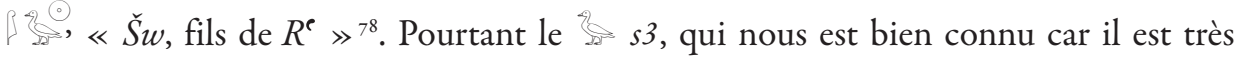
fréquent dans l'épigraphie égyptienne en raison de son sens « fils (de) », ne pourrait guère être remplacé, au hasard, par $m$ ou par ḥr. De plus, comment expliquer le

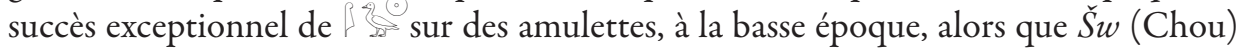
est un dieu issu du premier couple divin, ayant avant tout comme mission la séparation du ciel et de la terre ${ }^{79}$ ?

Reprenons un par un les hiéroglyphes inscrits sur notre scarabée : la présence du disque $\odot$ Aton, symbole de Rê, Soleil ou Démiurge, ne saurait guère nous surprendre sur un talisman ${ }^{80}$. Le faucon est probablement le motif le plus banal des portebonheur $^{81}$ : il est l'aspect animal d'Horus, le fils d'Isis et d'Osiris, premier souverain divin de l'Égypte et symbole, par excellence, de la divinité et de la monarchie égyptienne $^{8_{2}}$. La plume d'autruche est elle aussi un signe très puissant : on peut y voir l'emblème de Chou mais aussi de Maât, l'harmonie universelle qui permet au monde de vivre $^{8}$. Ainsi la réunion de ces trois symboles égyptiens ne pouvait, à notre sens, qu'être

proposées comme analogie par G. MatThiae SCandone, Scarabei e scaraboidi egiziani e egittizanti del Museo Nazionale di Cagliari, Roma 1975, B in, et F. Magnarini, Catalogo..., I0.55, qui acceptent également la lecture cryptographique présentée infra. W.M.Fl. PETRIE, Buttons..., p. 2I, affirme que ce motif est employé à partir de la $\mathrm{XX}^{\mathrm{c}}$ dynastie.

78 Traduction apud W.M.Fl. Petrie, Naucratis..., p. 37 (pour nos 83-84 et 85) ; P.E. Newberry, Egyptian Antiquities..., pl. XLI, no 9 ; W.M.Fl. Petrie, Buttons..., p. 2 I et n ${ }^{\text {os }}$ 708-709, 714 (pl. XII), I29I (pl. XVII); Chr. Blinkenberg, Lindos..., p. I403-I406 ; J. Vercoutter, Les objets égyptiens..., nº 206. Dans la base de données disponible sur le site Internet du Petrie Museum, on propose, plus vraisemblablement, « Maat, daughter of Ra », e.g. pour UC60778, qui ressemblerait également par sa forme et sa matière au scarabée d'Orgamè.

79 Ce qui le présente également comme, souffle divin, condition nécessaire à toute vie. Sur ses fonctions, cf., entre autres, les synthèses de Cl. Traunecker, s.u. dans DA; H. Velde, s.u. dans LÄ (1984). Pour une autre suggestion d'interprétation de cette inscription voir aussi L. BAQUÈs, Escarabeos Egipcios (Catalogo del Museo del Oriento biblico de Montserrat II), Ampurias (Barcelona) 1969-1970, pour le scarabée $\mathrm{n}^{\circ} 550101$.

80 En général, sur la religion solaire des Égyptiens, voir J. Assmann, Egyptian Solar Religion...

8I Cf. A. Grenfell, « Les divinités... », p. 8, in ; pour les faucons en faïence attribués aux ateliers de Rhodes, cf. Chr. Blinkenberg, Lindos ..., et V. Webb, Archaic Greek Faience ..., p. 92 (pour les exemplaires du Pont-Euxin).

82 Voir, pour une première approche, D. Valbelle, « Faucon », dans $D A$; Ph. Germond \& J. Livet, Bestiaire..., p. 132, I66; A. Altenmuller, « Falke dans $L \ddot{A}$ (1977). Pour plus de détails, voir J. Assmann, Re und Amun. Die Krise des polytheistischen Weltbilds im Ägypten der I8-20 Dynastie, Göttingen 1983, et, récemment, I. Guermeur, Les cultes d'Amon hors de Thèbes. Recherches de géographie religieuse, Brepols 2005 .

83 Malgré l'importance de cette déesse et des concepts qu'elle représente, il n'y avait pas de monographie à son sujet, avant la série de leçons données par J. Assmann, Maât. L'Égypte pharaonique et l'idée de 
perçue par les connaisseurs autrement qu'en tant que garantie de vitalité, de force, de pouvoir pour le porteur du scarabée.

Mais on peut aller plus loin dans la recherche du sens profond de cette série d'hiéroglyphes. C'est le mérite d'E. Drioton d'avoir trouvé la clé de ce type d'inscriptions : si sa théorie sur les « trigrammes d'Amon » a donné lieu à beaucoup de débats et de contestations de la part des savants, elle nous paraît néanmoins la seule explication cohérente que l'on ait jamais imaginée pour les bases trilittères de beaucoup de scarabées (comme le nôtre), surtout de l'époque qui nous concerne ${ }^{84}$. Partant du sens même du nom d'Amon, « Celui de qui le nom est caché », et des libertés qu'offrait l'écriture hiéroglyphique au scribe (c'est-à-dire de donner une nouvelle signification à un signe - par acrophonie, de changer l'ordre de l'écriture - par perturbation, et éventuellement de créer, sur les dos des scarabées, de petits tableaux - par « cryptographie thématique ${ }^{85}$ ), Drioton réalise un catalogue de hiéroglyphes qui auraient pu être utilisés dans l'écriture cachée du nom de ce dieu. Ce sont des «signes bénéfiques » qui « dans leur signification en clair [...] exprimaient des biens essentiels à la vie, et plus particulièrement à la vie heureuse, que tous les Égyptiens désiraient posséder ici-bas ou dans l'autre monde : la vie, la santé, la verdeur, le bien-être, la renommée, la souveraineté, la protection, le renouvellement, la perpétuité et d'autres privilèges encore dont nous saisissons mal la nature. [...] Utilisés, par jeu cryptographique, pour écrire le nom d'Amon, ces hiéroglyphes incorporaient ipso facto à ce nom la vertu de réaliser de tels biens $\gg$. Il nous reste donc à voir si les hiéroglyphes regroupés dans nos inscriptions correspondent aux phonèmes de 'Imn.

Le disque $\odot$ apparaît en deuxième position dans la liste de Drioton : il ne représente pas seulement le Soleil, mais aussi l'œil. On le lit donc comme $i$, (par acrophonie de l'im, « prunelle de l'œil », Erman \& Grapow I 75, 23), comme $m$ (de $m 33$, « ce qui voit », Erman \& Grapow II, 7), mais aussi comme $n$, représentant une graphie de $\otimes$.

\footnotetext{
justice sociale (Leçons Collège de France 1988), Paris 1989 ; plus récemment, $c f$. E. TeETER, The Presentation of Maat. Ritual and Legitimacy in Ancient Egypt, Chicago 1997. C'est la plume de Maât que l'on met en balance avec l'âme du mort, lors de la psychostasie.

84 Cf. E. Drioton, « Trigrammes d'Amon », Wiener Zeitschrift für die Kunde des Morgenlandes 54, Festschrift Hermann Junker zum 80. Geburtstag gewidmet von seinen Freunden und Schülern, 1957, p. II-33, qui invente la dénomination « trigramme » et l'explique : «On entend ici par trigrammes d'Amon des expressions du nom d'Amon au moyen de trois signes unilittères, empruntés au syllabaire cryptographique. Le mot est choisi pour évoquer, par analogie, la doctrine des cabalistes juifs qui faisaient de l'écriture en quatre lettres du nom sacrosaint de Yahweh, ou tétragramme, un talisman redoutable ».

85 Voir également les études de Drioton republiées dans E. Drioton \& H.W. Fairman, Cryptographie ou pages sur le développement de l'alphabet en Égypte ancienne, éd. Dr DiA' Aвоu - GHAZI, Le Caire 1992.
} 
Le faucon peut être lu comme $i$ (de ` $\underline{h} m>i \underline{h} m$ « faucon », Erman \& Grapow I 226, 6), comme $m$ (de $M n t w$, le dieu Month/Montou, Erman \& Grapow II 92, 2) et comme $n$ (de $n \underline{t} r$, « dieu », Erman \& Grapow II 358). P peut valoir $i$ (par « équivalence groupale » avec ) aussi bien que $m$, par acrophonie de $m 3^{e}$, « juste » (Erman \& Grapow II I2). Mais, si à la place du faucon on retrouve la chouette nous sommes obligée de considérer qu'il s'agit d'un $m$ : la chouette, animal maléfique, ne serait autrement jamais marquée sur un porte-bonheur ${ }^{86}$. Quant au 3 , ce n'est qu'une autre manière d'écrire le même $m$, par acrophonie pour $m s ̌ j . t$, « oiseau aquatique » (Erman \& Grapow II I 43, $3)^{87}$. Dans ces conditions, les signes deviennent interchangeables et les détails morphologiques, qui permettraient leur identification précise, facultatifs.

Ainsi lisons-nous sur les bases des scarabées d'Orgamè, d'Olbia, de Naucratis et de Carthage :

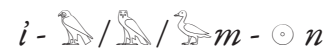

le nom d'Amon, seigneur des dieux et de tout ce qui existe, donneur de souffle à tout ce qui naît. Rappelons, à titre d'exemple pour les fonctions du dieu de Thèbes, quelques lignes traduites d'une prière privée :

Heureux celui qui voit Amon-Rê ! Amon conduit au bonheur. Amon-Rê procure ce qui est bon. Amon-Rê donne la prospérité. Celui qu'Amon protège vivra. Amon-Rê est la force de l'isolé. Amon-Rê est le refuge de l'affligé. ${ }^{88}$

Pourtant, si autant de savants égyptologues modernes n'ont pas vu ou refusent encore de voir le nom d'Amon sur ces scarabées ${ }^{89}$, qu'en reste-t-il des Grecs qui ont porté ces amulettes à partir du VIII ${ }^{e}$ s. av. J.-C., lesquels ne connaissaient pas les

86 Cf. A. Grenfell, « Les divinités... », p. I4. Sur ce hiéroglyphe, avec plus de détails, voir l'explication de P. LACAU, « Le signe [...] m », BIFAO 69, 1971, p. 239-243.

87 Sur le symbole de l'oie, animal d'Amon, voir, entre autres, Ch. Kuentz, « L'oie du Nil (Chenalopex Aegyptiaca) dans l'Antique Égypte », Archives du Muséum d'histoire naturelle de Lyon, 1934, p. I-64 ; Ph. Germond \& J. Livet, Bestiaire..., p. 122.

$88 C f$. E. Drioton, « La dévotion privée à l'époque pharaonique », Cahiers d'histoire égyptienne, série I, fasc. 4, Héliopolis 1949, p. 295-305.

89 Voir, parmi ceux qui acceptent les «trigrammes » de Drioton, les réserves de H. Poncy et alii, «Sceaux du musée d'Adana... », Anatolia antiqual Eski Anadolu 9, 2001, p. 9-37, surtout p. 21 sq. qui se demandent «s'il faut systématiquement interpréter la gravure des scarabées, susceptibles d'être non égyptiens - distinction que l'on ne peut assurer avec certitude - en terme de valeurs cryptographiques tant la symbolique égyptienne devait être imparfaitement, voire nullement, comprise en Anatolie, à Chypre et au Levant, autant de régions où ont été largement fabriqués et ont circulé les scarabées égyptisants ». 
hiéroglyphes et ne croyaient pas en Amon ${ }^{\circ 0}$ ? À notre sens, il faudrait imaginer l'usage des scarabées égyptiens en terre grecque ou en terre barbare grécisée comme, encore de nos jours, le port des amulettes à marques occultes : on ne connait pas forcément les sens et l'histoire des signes, mais on est capable de reconnaître qu' il s'agit d'un talisman qui prêterait toute sa force vitale à celui qui le possède.

Revenons aux bouches du Danube, dans la maison orgaménienne située à l'extrémité de l'espace fortifié, à la fin du $\mathrm{IV}^{\mathrm{e}} \mathrm{s}$. av. J.-C. : les deux scarabées retrouvés dans la seule pièce conservée de cette maison font partie des productions massives, « en série », des ateliers (probablement) du Delta égyptien qui, comme celui fouillé par Petrie à Naucratis, travaillaient pour l'exportation sur les marchés égyptien, grec, étrusque, punique ou barbare. Il nous est aujourd'hui impossible de préciser la localisation géographique de ces fabriques : seuls l'essor des analyses chimiques et leur association permanente à l'étude stylistique des scarabées et des moules pourraient apporter quelque précision supplémentaire dans cette recherche. Nous ignorons, hélas, sans doute à jamais, l'origine ethnique (égyptienne, phénicienne, grecque etc.) des artisans qui manufacturaient ces objets. La faience n'était ni un secret ni un monopole égyptien. Sa technique était déjà connue, depuis quelques milliers d'années, en Orient ; au VIII ${ }^{e}$ s. av. J.-C. (et ceci en laissant de côté la production « isolée » chronologiquement de l'Âge du Bronze), des vases en faïence étaient déjà produits en Grèce. Même la forme du scarabée était devenue, à l'époque archaïque, le modèle principal des entailles phéniciennes et grecques. Les hiéroglyphes inscrits sur les scarabées ne pourraient guère être pris comme témoins dans ce débat : bien ou mal tracés, ils pouvaient être estampés aussi bien par une main égyptienne que par une main grecque. Incisé, leur trait dépend du sens artistique de l'artisan, lequel les reproduit à partir d'un modèle, plutôt que de sa compréhension de l'écrit ; de plus, même si le bénéficiaire ressenti était égyptien, rien ne nous assure que celui-ci eût compris la valeur des signes inscrits sur une amulette.

Les artisans faïenciers pouvaient s'inspirer, dans la création de leurs moules, d'une tradition millénaire qui avait tout mis en œuvre pour représenter le Scarabaeus Sacer et dont nous ne possédons plus que des bribes. Les chercheurs modernes ont essayé d'identifier des tendances dans la figuration de l'insecte : un scarabée « médiocre » proviendrait plutôt de l'époque tardive. Il suffit cependant de regarder la forme des scarabées en faïence égyptienne d'époque romaine pour voir qu'ils n'auraient pu être dérivés d'une représentation aussi simplifiée que celle de notre deuxième scarabée. Nous devrions donc prendre en compte l'existence de plusieurs ateliers à la même époque

90 Sur l'identification Amon-Zeus (Hérodote 2.42) et les autres témoignages littéraires grecs ainsi que sur le culte d'Amon à Naucratis, $c f$. I. Guermeur, Les cultes d'Amon.... 
voire la possibilité qu'un atelier produisît des objets de plusieurs types et qualités en même temps ${ }^{91}$.

Devant cet amalgame des formes (de surcroît compliqué par la spécificité des matériaux dans lesquels ont été réalisés les scarabées), les savants ont essayé, dans un premier temps, de préciser leurs typologies d'après le décor incisé, ultérieurement d'après le corps de l'insecte et finalement en combinant toutes ces variables ${ }^{92}$. Néanmoins, comme les publications disponibles ne donnent généralement que le dessin de la base, les analogies que nous proposons ne sont guère concluantes : les symboles inscrits sur ces talismans sont reproduits à l'identique dans plusieurs ateliers, pendant plusieurs générations ou, s'il l'on prend l'exemple du Tilapia, pendant plusieurs dizaines de générations. Si les scarabées d'Olbia et de Carthage présentent des trigrammes similaires à celui d'Orgamè, cela ne signifie pas qu'ils ont été fabriqués au même endroit ou à la même époque : tout ce que ces exemples nous apprennent c'est que cette combinaison cryptographique était utilisée là où ont été créées les amulettes enfouies à Olbia et à Carthage au VI' s. av. J.-C.

Souvent, l'accord entre la datation du contexte archéologique de la découverte et celle proposée par les analogies n'est pas facile à établir ${ }^{93}$ : si pour les très nombreuses trouvailles de Perachora, Lindos, Ialyssos ${ }^{94}$, datées de plus d'une centaine d'années avant la fondation de l'atelier de Naucratis, auquel elles avaient été attribuées, les spécialistes ont fini par être obligés de reconnaître une autre originess, pour plusieurs des types puniques de Gorton, provenant de contextes datés jusqu’à mille ans plus tard que leurs analogies orientales, le chercheur a le choix d'imaginer la transmission de ces pièces de génération en génération (pratique d'ailleurs attestée dans l'Égypte pharaonique) ou

9I Les moules de scarabées mis au jour par Petrie à Naucratis, $c f . s u p r a$, pourraient constituer une preuve dans ce sens.

92 Cf. les travaux de Ward et alii; A.F. GorTon, Egyptian and Egyptianizing Scarabs..., avec les critiques de E. Teeter, « (Review) Egyptian and Egyptianizing Scarabs. A Typology of Steatite, Faience and Paste Scarabs from Punic and Other Mediterranean Sites », AJA I03.I, 1999, p. I40-I 4I.

93 Les contextes ambigus des fouilles, légales et illégales, du XIX ${ }^{\mathrm{e}}$ s. posent d'autant plus de problèmes : e.g. le scarabée d'Olbia, publié par B. TouraefF, « Objets... », 23 (fig. 6), parmi les « objets de ma collection qui ont été acquis à Odessa comme provenant d'Olbia », représentant un «Scarabée de dimensions considérables $(3 \mathrm{~cm} \mathrm{x} 2 \mathrm{~cm})$ avec la représentation du scarabée volant. Terre émaillée verte » appartient au groupe de scarabées Hyksos (A.F. GorTon, Egyptian and Egyptianizing Scarabs..., type V, dont très peu d'exemplaires ont été retrouvés dans des contextes aussi tardifs que le ${ }^{\mathrm{Ir}}$ millénaire av. J.-C.).

94 T.J. Dunbabin éd., Perachora..., ce site grec ayant fourni le plus grand nombre de scarabées ; Chr. Blinkenberg, Lindos... ; G. Jacopi, Clara Rhodos. Studi e materiali pubblicati a cura dell'Istituto storico-archeologico di Rodi. Vol. III Scavi nella necropoli di Jalisso, Rodi 1939, pour la nécropole de Ialyssos. 95 T.J. Dunbabin éd., Perachora..., p. 46i-5i6. 
d'accepter une production ayant continué au-delà des limites chronologiques prédéterminées. Les scarabées d'Orgamè nous posent un problème similaire : comment concilier le contexte archéologique (qui prouve la contemporanéité de ces objets et des céramiques du dernier quart du $\mathrm{IV}^{\mathrm{e}} \mathrm{s}$. av. J.-C) avec la date des analogies proposées (début $\mathrm{VI}^{\mathrm{e}}$ s. - milieu $\mathrm{V}^{\mathrm{e}}$ s. av. J.-C., d'après la datation générale des nécropoles de Carthage et d'Olbia) ? Rappelons aussi que tous les autres scarabées découverts autour de la mer Noire, comparables à ceux d'Orgamè (autrement dit, assimilés au type « naucratite ») proviennent de contextes datés du VI ${ }^{\mathrm{e}}$ s. av. J.-C. ${ }^{96}$

L'hypothèse d'une fabrication et d'une importation de ces objets pendant le $\mathrm{VI}^{\mathrm{e}}-\mathrm{V}^{\mathrm{e}}$ s. av. J.-C. semble recevable : les scarabées auraient pu venir de Naucratis, peutêtre indirectement, par exemple par l'intermédiaire de Chios, de Rhodes, de Samos, de Clazomène, toutes métropoles de l'emporion égyptien ${ }^{97}$. En effet, Orgamè, tout comme Bérézan et Olbia, a révélé des céramiques archaïques certifiant des contacts commerciaux (directs ou par d'autres intermédiaires) avec ces centres de la Grèce de l'Est $^{98}$. De plus, la découverte d'un aryballe fragmentaire, en faïence turquoise, peutêtre « naucratite », à Vișina (dans le territoire d'Orgamè), dans un contexte de la fin $\mathrm{du} \mathrm{VI}^{\mathrm{e}}$ et du début du $\mathrm{V}^{\mathrm{e}}$ s. av. J.-C.99, conforte l'idée que cette région était intégrée à

96 Outre les scarabées inventoriés par E.M. Алексеева, Античныц бусы..., 1975, p. 23-49, pl. 7-12 (dont les analogies citées plus haut, d'Olbia), mentionnons les découvertes de Kertch et de Taman, $c f$. Б.Б. Пиотровский, « Аревнеегипетские предметы ... » (qui organise les objets « égyptiens » dans trois catégories - importations archaïques et classiques, hellénistiques et romaines - et les inscrit sur une

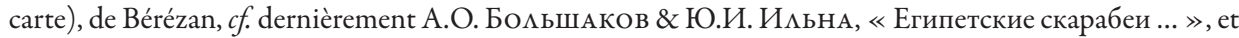
le scarabée « de l'époque saïte », aujourd'hui perdu, découvert à Histria, dans la Zone Sacrée, « au temple d'Aphrodite' » (lequel s'est avéré être le temple de Zeus), avec « un petit dieu Bès en terre cuite » et un fragment d'aryballe en faïence, daté au deuxième quart du VI ${ }^{\mathrm{e}}$ s. av. J.-C. (cf. M. LAmbrino, Les Vases archaïques d'Histria, Bucureşti 1938, p. 34 sq. ; C. DomăneAnȚU, « Archaic Greek Faience Imports in the Black Sea Area », Dacia N.S., 32.I-2, 1988, p. 21-25, surtout p. 21).

97 Cf. J. Yoyотте, « Naucratis, ville égyptienne », Annuaire du Collège de France 92,1991-1992, p. 634-644, « Les contacts entre Égyptiens et Grecs (VII ${ }^{\mathrm{e}}$-II ${ }^{\mathrm{e}}$ siècles av. J.-C.) », Annuaire du Collège de France 94, 1993-1994, p. 679-698, et « Les contacts entre Égyptiens et Grecs (VII ${ }^{\mathrm{c}}-\mathrm{II}^{\mathrm{c}}$ siècles av. J.-C.) 》, Annuaire du Collège de France 95, 1994-1995, p. 669-683 ; A. MöLler, Naukratis... ; sur les contacts directs et indirects entre le monde grec et l'Égypte, voir encore la synthèse historique de M.M. Austin, Greece and Egypt in the Archaic Age, PCPhS Suppl. 2, 1970, p. 35 sq., et, dans une perspective archéologique, les études réunies par A. Villing \& U. Schlotzhauer (éd.), Naukratis : Greek Diversity in Egypt. Studies on East Greek Pottery and exchange in the Eastern Mediterranean, London, 2006.

98 Cf. M. Mănucu-Adameşteanu, Céramique archaïque d'Orgamè, Thèse, Bucarest 1999 ; S.D. KRYZHYTSKYY \& alii, « Olbia... », avec bibliographie.

99 Cf. M. Mănucu-Adameşteanu, « Sondajul efectuat în aşezarea antică de la Vişina, com. Jurilovca, jud. Tulcea », $M C A$, 1980, p. 157-160; C. DomĂnEAnȚU, « Archaic Greek Faience... », p. 23. Sur 
ce réseau d'échanges. Ainsi, conservés pendant plusieurs générations, les deux scarabées auront-ils « survécu » de manière continue jusque vers la fin du IV ${ }^{\mathrm{e}}$ s. av. J.-C.

Pourtant cette explication ne nous paraît guère exclusive. Même si l'on soutient généralement que la production de scarabées en faïence aurait cessé avec l'invasion perse $^{100}$ et que les relations, directes et indirectes, entre Naucratis et la mer Noire se seraient arrêtées à ce moment-là ${ }^{101}$, les découvertes archéologiques pourraient désormais nuancer ces positions. Tout d'abord, comme l'ont montré les fouilles archéologiques successives, au $\mathrm{IV}^{\mathrm{e}}$ s. av. J.-C., Naucratis est une ville fleurissante ${ }^{102}$. La présence des timbres amphoriques thasiens prouve incontestablement un lien commercial entre l'extrémité septentrionale de l'Égée et l'Égypte ${ }^{103}$. Le célèbre vin thasien est alors demandé aussi bien à Naucratis qu'à Orgamè, où les restes amphoriques issus des fouilles sont très significatifs ${ }^{104}$. De plus, les dernières recherches qui ont inventorié les preuves des échanges entre le monde grec et le monde perse, ont considérablement tempéré le préjugé d'une séparation nette entre les deux voisins ${ }^{105}$. Quant aux artefacts

les recherches menées dans cet établissement de la chora d'Orgamè, $c f$. M. MănUCU-AdameşTEAnU, « Orgame», p. 352 .

ioo Cf. e.g. W.M.Fl. Petrie, Scarabs..., p. 33. Voir plus récemment la position ferme de J. Padro I ParCeRISA, Egyptian-Type Documents from the Mediterranean Littoral of the Iberian Peninsula before the Roman Conquest, Leiden 1985 , I, p. 55-56: «With very rare exceptions, the glazed composition scarabs [...] are of Egyptian manufacture and their dating cannot in any instance be brought lower than the $6^{\text {th }}$ century. $\gg$ Aussi II, 2I. Il accepte néanmoins (II, Empuries $n^{\circ}$ 07.17) que «In the $4^{\text {th }}$ century amulets undeniably Egyptian both in technique and workmanship continue to appear in Carthage. In Egypt itself the quality of small amulets is very mediocre in this period, as I have already noted above. Finally there is no formal evidence that the glazed composition technique was known outside Egypt ».

Iо $C f$. la thèse de A.О. БольшАКов \& Ю.И. ИАьнА, « Египетские скарабеи ... », qui datent les scarabées de Bérézan entre le milieu et le troisième quart du $\mathrm{VI}^{\mathrm{c}} \mathrm{s}$. av. J.-C., donc avant 525 av. J.-C. Le même terminus ante quem est argumenté par C. DOMĂNEANȚU, « Archaic Greek Faience... », pour les aryballes en faïences découverts à Histria (cinq), Tariverde (un) et Vişina (un) ainsi que pour toutes les autres découvertes qu'elle a pu inventorier à Bérézan, Olbia et Panticapée.

IO2 Déjà E.A. GARdner, Naucratis..., p. 26-27, remarquait lors des fouilles dans la nécropole que les tombes du IV ${ }^{e}-I_{I I}{ }^{e}$ s. av. J.-C. étaient les plus riches.

103 Cf. W.D.E. Coulson et alii, Ancient Naukratis vol II. The Survey at Naukratis and Environs, Part I. The Survey at Naukratis, Oxford 1996, p I47 sq., n ${ }^{\circ s}$ 2I-23 (dont le plus ancien, $n^{\circ}$ 22, est daté de la XX dynastie, 404-34I av. J.-C.).

IO4 $C f$. M. MănUCU-Adameşteanu, « Orgame », p. 370, avec bibliographie.

Ios Voir M.C. Miller, Athens and Persia in the Fifth Century BC. A study in cultural receptivity, Cambridge 1997, sur la mobilité des objets (butin de guerre ou commerce). Voir aussi la thèse de P.G. vaN ALfeN, Pant'agatha. Commodities in Levantine-Aegean Trade during the Persian Period, 6-4th c. BC, University of Texas 2002 (inédite), qui dresse la liste des produits de luxe (aliments, colorants, minéraux et métaux 
toreutiques achéménides identifiés depuis longtemps en Thrace, ils sont datés précisément de la deuxième moitié du IV ${ }^{\mathrm{e}}$ s. av. J.-C. ${ }^{106}$

En second lieu, nous devons prendre en compte la situation des amulettes importées à Carthage : comme l'a remarqué Vercoutter, à partir du $V^{\mathrm{e}}$ s., le nombre de scarabées en faïence égyptienne est en baisse, mais ces scarabées ne disparaittront pas avant la fin du $\mathrm{III}^{e}$ s. av. J.-C. Au moins une partie de ces objets auraient pu être produits pendant et même après le $\mathrm{V}^{\mathrm{e}} \mathrm{s}$. av. J.-C., bien qu'à partir de cette date on leur préfère, de plus en plus, les « scarabées puniques ».

C'est un phénomène similaire que nous estimons apercevoir sur les rives de la mer Noire. Des talismans en faïence égyptienne ont été importés à partir $d u\left(V_{I I}{ }^{\mathrm{e}}-\mathrm{VII}^{\mathrm{e}} \mathrm{s}\right.$. av. J.-C. à Bérézan, Olbia, Chersonèse ${ }^{107}$, Néapolis ${ }^{108}$, Panticapée, etc en provenance du Delta. ${ }^{109}$. Au V $V^{e}-I V^{e}$ s. av. J.-C., ces objets deviennent plus rares ; ils entrent, comme ceux de Carthage, en concurrence avec d'autres types d'amulettes qui finiront par en prendre le dessus. Au-delà des changements politiques et économiques imposés par les Perses, l'on aurait affaire aussi à un phénomène de mode : les scarabées en métaux et en pierres (semi)précieuses, décorés avec des thèmes grecs ${ }^{10}$ ainsi que les perles/pendentifs

de tout genre et sous toutes les formes, animaux, esclaves, artefacts en différentes matières) échangés. On attend encore une première étude sur les exportations d'Égypte à cette époque.

Io6 Cf. P. Alexandrescu, « MHAIZEIN. À propos des importations et de l'influence achéménide en Thrace », Dacia N.S. 30.I-2, 1986, p. I55-158, et « L'Atelier Agighiol et l'Iran pré-achéménide », Il Mar Nero I, 1995-1996, p. 9-27.

I07 Cf. le pendentif en forme de tête de bélier, en verre et en or, découvert à Chersonèse et interprété par В.П. Алексевв, « Новые материалы к изучению культуры античных городов Северного Причерноморья (Тира - Никоний - Омьвия - Херсонес) (New Materials for the Cultural Study of the Ancient North Pontic Cities [Tyras - Niconium - Olbia - Chersonesus]) », VDI 250.3, 2004, p. 58-79, surtout p. 78-79, comme une production de Naucratis, du VI' s. av. J.-C.

Io8 E.g. le scarabée en « jaspe vernissé », bien conservé dans sa bague en or, présenté lors de l'exposition « L'Or des Scythes » (E.D. REeDER dir., L'or des rois scythes, Catalogue d'exposition, Grand-Palais Paris 25 septembre-31 décembre 2001, n 60, et Dossiers d'Archéologie 266, p. 12).

I०9 Nous espérons réunir bientôt dans un catalogue tous les scarabées «égyptiens », en provenance de la mer Noire, mentionnés dans les publications.

Iо Cf. О. Неверов, « Етрусские скарабеи из Северного Причерноморья », $S G E$ 40, 1975, p. 38-42, et, d'une manière générale, ses ouvrages sur les entailles antiques ; pour l'usage comme sceaux, voir son article «Impressions of Seals on Northern Pontic Pottery », dans Northern Pontic Antiquities in the State Hermitage Museum, éds J. Boardman \& S.I. Solovyov \& G.R. Tsetskhladze, Leiden 200 I (avec bibliographie). Des images d'objets de ce type ont été publiées en couleurs dans E.D. ReEDer, L'Or... ( $\mathrm{n}^{\text {os }} 68$ en cornaline avec monture d'or, 98 et 99 en or). 
en verre polychrome, d'aspect zoo- et anthropomorphe, dites «phénicien(ne)s » ${ }^{\mathrm{II}}$, auront peut-être mieux répondu au goût de l'époque.

Cette tendance sera inversée dès la fin du IV ${ }^{e}$ et surtout le début du $\mathrm{III}^{\mathrm{e}} \mathrm{s}$., une fois que Ptolémée II aura consolidé le pouvoir de l'Égypte dans le bassin égéen. Nombreux sont les témoignages historiques et archéologiques des contacts entre le royaume ptolémaïque et les Grecs de la mer Noire : il suffit de rappeler l'expédition du navire Isis ${ }^{\mathrm{II}}$, la diffusion des cultes égyptiens ${ }^{113}$ et, avec eux, de nouveaux talismans ${ }^{114}$, les découvertes céramiques $^{115}$. Réciproquement, des citoyens du Pont-Euxin se rendent en Égypte ${ }^{116}$ et des produits pontiques arrivent sur les marchés égyptiens ${ }^{117}$.

Ainsi, quelle que soit la date de leur fabrication (entre le VI ${ }^{\mathrm{e}}$ et le $\mathrm{IV}^{\mathrm{e}} \mathrm{s}$. av. J.-C.), les deux scarabées d'Orgamè peuvent être regardés, en raison du contexte dans lequel ils ont fini leur existence antique, comme un lien historique entre l'archaïsme (auquel

III Nous pensons, en premier lieu, aux perles en verre anthropomorphes, dites « phéniciennes », qui ont connu au $\mathrm{IV}^{\mathrm{c}}$ s. av. J.-C. un succès remarquable dans la région pontique. Cf. E.M. A бусы Северного Причерноморья, Москва 1978 et 1982 ; M. SEEFRIED, Les Pendentifs en verre sur noyau des pays de la Méditerranée antique, Rome 1982 ; J. Bouzek, « Les Phéniciens... ».

II2 Cf. Ju.G. Vinogradov, « Der Staatsbesuch der 'Isis' im Bosporos », ACSS 5.4, 1999, p. 271-302 ; O. Höckmann, « Naval and Other Graffiti from Nymphaion », ACSS 5.4, 1999, p. 303-356 ; W.M. MurraY, « A Trireme Named Isis : the Sgraffito from Nymphaion », IJNA 30.2, 2001, p. 250-56 (avec bibliographies).

II3 Cf. M.M. Kobylina, Divinités orientales sur le littoral nord de la mer Noire. Appendice O.J. NévéROv, Leiden 1976 ; M. TACHeVA, Eastern Cults in Moesia Inferior and Thracia, Leiden 1983 ; Ju.G. VINoGRADOV \& M.I. Zolotarev, « Worship of the Sacred Egyptian Triad in Chersonesus (Crimea) », $A C S S$ 5.4, 1999, p. 357-381.

II 4 Voir l'inventaire dressé par M.-D. NENNA \& M. SEIF EL DIN, « La petite plastique en faïence d'Alexan-

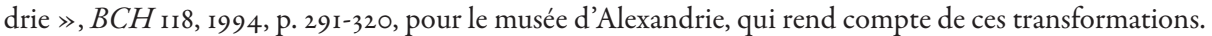

in Cf., sur le vase de faïence ptolémaïque d'Histria, P. AleXANDresCU, « Însemnări arheologice. Un vas de faianță ptolemaic la Histria », StCl 26, 1988, p. II6-121, «Un vase ptolémaïque en faïence d'Istros » dans Festschrift für Nikolaus Himmelmann, éds H.-U. Cain \& H. Gabelmann \& D. Salzmann, Mainz 1989, p. 305-309, et Histria VII. La Zone Sacrée d'époque grecque, Bucureşti-Paris 2005, p. 412-413; pour les céramiques Hadra, $c f$. V. Lungu, « Céramique du style Hadra à Histria », Il Mar Nero 4, 1999-2000, p. $43-87$.

II6 Cf. L. Ruscu, Relațiile externe ale oraşelor grecești de pe litoralul românesc al mării Negre, Cluj 2002, p. 227-230. La prosopographie externe des citoyens pontiques, préparée actuellement par M. Alexandru Avram (qui a généreusement mis à ma disposition son manuscrit), offrira un tableau complet de ces déplacements ; voir, pour l'instant, A. Avram, « L'Égypte lagide et la mer Noire : approche prosopographique », dans La Méditerranée d'une rive à l'autre: culture classique et cultures périphériques. Actes, Cahiers de la Villa « Kérylos » n i8, éds A. Laronde \& J. Leclant , Paris 2007, p. I27-153.

II7 Voir, e.g. pour le miel et la cire, les témoignages papyrologiques et littéraires rassemblés par H. Chouliara-Raïos, L'Abeille et le miel en Égypte d'après les papyrus grecs, Ioannina 1989, p. 74 sq., 165-166. 
sont généralement rattachées les faïences de leur type) et l'époque hellénistique (qui vit les échanges de toute sorte s'épanouir entre le Pont et l'Égypte). Découvertes dans une maison du dernier quart du $I^{\mathrm{e}} \mathrm{s}$. av. J.-C., près de l'enceinte classique, ces deux amulettes en céramique couverte de glaçure verdâtre, façonnées d'après la forme, plus ou moins simplifiée, du Scarabaeus Sacer, portent sur leurs bases des symboles apotropaïques : la carpe du Nil, Tilapia Nilotica, un des poissons les plus fréquents dans l'art égyptien, reconnaissable par la forme de ses nageoires, est le signe de la résurrection. Il répand sa descendance de sa bouche, comme l'avait fait le Créateur du monde ; il laisse entrevoir ses reflets rougeâtres dans les ondes matinales du Nil comme le Soleil et comme le défunt qui prendront sa forme avant de renaître du « fleuve de turquoise ». Vainqueur du monstre des ténèbres qui avait mis en danger le retour diurne du Soleil, Tilapia garde dans sa bouche la fleur qui s'était ouverte au début du monde et de laquelle est né le soleil même : tel Osiris qui ressort du Nil pour une nouvelle vie. Tout aussi riche en significations est le nom en « écriture cachée » du dieu « au nom caché », Amon : ingénieux le scribe qui a voulu donner au dieu des dieux, protecteur de celui qui portera le scarabée, la justice de Maât, la majesté de Horus, la vie de Rê ! Ce sont des vœux répétés d'innombrables fois, pendant des générations, sur des scarabées découverts sur tout le pourtour de la Méditerranée, d'Égypte et d'Asie Occidentale jusqu'à Carthage et au NO de la mer Noire. Deux mille cinq cents ans après, ils existent toujours et font « revenir à l'existence », par leur message, ceux à qui ils promettaient la vie. 